\title{
Gram-scale, Cheap, and Eco-friendly Iron-catalyzed Cross-Coupling between Alkyl Grignard reagents and Alkenyl or Aryl halides.
}

\author{
Supporting Information
}

Gérard Cahiez, ${ }^{* \dagger} \uparrow$ Guillaume Lefèvre, ${ }^{*,}$ Alban Moyeux, ${ }^{,}$Olivier Guerret, $\square$ Eric Gayon, ${ }^{\square}$ Loïc Guillonneau, ${ }^{\square}$ Nicolas Lefèvre, ${ }^{\dagger, a}$ Qinzhuo Gu, ${ }^{\dagger}$ and Edouard Zhou ${ }^{\dagger, \downarrow, \square}$

$\dagger$ Institut de Recherche de Chimie Paris, CNRS (UMR 8247) - Chimie ParisTech, PSL Research University, 11 rue Pierre et Marie Curie, 75005 Paris, France

$¥$ Chimie ParisTech, PSL University, CNRS, Institute of Chemistry for Life and Health Sciences, CSB2D, 75005 Paris,

France

${ }^{\circledR}$ Laboratoire CSPBAT - UMR 7244 - Université Paris 13, 1 rue de Chablis, 93017 Bobigny Cedex, France

$\square$ M2i Development, Bâtiment ChemStart’Up, Allée Le Corbusier, 64170 Lacq, France

a M2i Salin, 36 Route d'Arles RD 36, 13129 Salin de Giraud

\section{Table of contents}

$\begin{array}{ll}\text { Instrumentation and Chemicals } & \text { S-2 }\end{array}$

Synthesis of starting materials $\quad$ S-3

Table S1: Optimization of the concentration for alkyl-vinyl cross-coupling reaction $\quad$ S-4

Table S2: Optimization of the temperature for alkyl-vinyl cross-coupling reaction $\quad$ S-4

Table S3: Optimization of the nature of the RO[M] additive for alkyl-aryl cross-coupling reaction

Table S4: Optimization of the quantity of $\mathrm{FeCl}_{3}$ for alkyl-aryl cross-coupling reaction $\quad$ S-5

Table S5: Optimization of the quantity of EtOMgCl for alkyl-aryl cross-coupling reaction S-5

Table S6: Optimization of the temperature for alkyl-aryl cross-coupling reaction S-6

Table S7: Optimization of the concentration for alkyl-aryl cross-coupling reaction $\quad$ S-6

$\begin{array}{ll}\text { General procedures for cross-coupling reactions } & \text { S-7 }\end{array}$

Characterization Data $\quad$ S-8

$\begin{array}{ll}\text { Bibliography } & \text { S-15 }\end{array}$

${ }^{1} \mathrm{H}$ and ${ }^{13} \mathrm{C}$ NMR spectra of synthesized compounds $\quad$ S-16 


\section{Instrumentation and Chemicals}

Analyses

Gas chromatography analyses (GC) were performed on two different chromatographs. The first one was a Shimadzu Chromatograph 2010 Plus apparatus (GC2010) equipped with a flame ionization detector. The capillary column was a Zebron ZB-5MS (length: $10 \mathrm{~m}$, I.D.: $0.10 \mathrm{~mm}$, film thickness: $0.10 \mu \mathrm{m} .5 \%$ polysilarylene / 95\% polydimethylsiloxane). The second one was a Shimadzu Chromatograph 2014 Plus apparatus (GC2014) equipped with a flame ionization detector. The capillary column was a Zebron ZB-35 (length: $10 \mathrm{~m}$, I.D.: $0.10 \mathrm{~mm}$, film thickness: $0.10 \mu \mathrm{m} .35 \%$ phenyl / 65\% polydimethylsiloxane). Hydrogen was used as a carrier gaz $(1.14 \mathrm{~mL} / \mathrm{min}$, ratio split: 80). Three standard analysis conditions: $40{ }^{\circ} \mathrm{C}$ (hold $1 \mathrm{~min}$ ) to $200{ }^{\circ} \mathrm{C}$ (hold $3.5 \mathrm{~min}$ ), heating rate: 30 ${ }^{\circ} \mathrm{C} / \min$ (Method 1); $80{ }^{\circ} \mathrm{C}$ (hold $0 \mathrm{~min}$ ) to $200{ }^{\circ} \mathrm{C}$ (hold $4 \mathrm{~min}$ ), heating rate: $20{ }^{\circ} \mathrm{C} / \min ($ Method 2) ; $80^{\circ} \mathrm{C}$ (hold $0 \mathrm{~min}$ ) to $250^{\circ} \mathrm{C}$ (hold $10 \mathrm{~min}$ ), heating rate: $20^{\circ} \mathrm{C} / \mathrm{min}$ (Method 3).

Mass spectra were recorded on a Hewlett-Packart HP 5973 mass spectrometer via a GC/MS coupling with a Hewlett-Packart HP 6890 chromatograph equipped with a capillary column HP-5MS (50 m x $0.25 \mathrm{~mm} \times 0.25 \mu \mathrm{m}$ ). Ionisation was performed by electronic impact (EI, $70 \mathrm{eV}$ ). Mass spectra are reported as $\mathrm{m} / \mathrm{z}$ (molecular ion peak, \% of relative intensity).

Thin layer chromatography (TLC) was carried out on silica gel plates (Carlo Erba 60 F254). Spots were detected with UV light.

Flash chromatography was performed on silica gel columns (Carlo Erba, spherical, neutral, 40-60 $\mu \mathrm{m}$ ). ${ }^{1} \mathrm{H}$ NMR $\left(300 \mathrm{MHz}, \mathrm{CDCl}_{3}\right),{ }^{13} \mathrm{C}$ NMR $\left(75 \mathrm{MHz}, \mathrm{CDCl}_{3}\right.$ ) and ${ }^{19} \mathrm{~F}$ NMR $\left(282 \mathrm{MHz}, \mathrm{CDCl}_{3}\right.$ ) were recorded on a Brucker Avance III HD 400 instrument. Chemical shifts ( $\delta$ ) are given in ppm from TMS $\left({ }^{1} \mathrm{H},{ }^{13} \mathrm{C}\right)$ and Trichlorofluoromethane $\left({ }^{19} \mathrm{~F}\right)$. Coupling constants $(J)$ are given in Hz. The following abbreviations are used: $s$, singlet, $d$, doublet, $t$, triplet, $q$, quartet, $m$, multiplet, $d m$, multiplet of doublet.

Mass spectra were recorded on a Hewlett-Packart HP 5973 mass spectrometer via a GC/MS coupling with a Hewlett-Packart HP 6890 chromatograph equipped with a capillary column HP-5MS (50 m x $0.25 \mathrm{~mm} \times 0.25 \mu \mathrm{m})$. Ionisation was performed by electronic impact (EI, $70 \mathrm{eV}$ ). Mass spectra are reported as $\mathrm{m} / \mathrm{z}$ (\% of relative intensity).

ESI-MS experiments were carried out using a LTQ-Orbitrap XL from Thermo Scientific (Thermo Fisher Scientific, Courtaboeuf, France) and operated in positive ionization mode, with a spray voltage at $3.6 \mathrm{kV}$. Sheath and auxiliary gas were set at a flow rate of 45 and 15 arbitrary units (a.u.), respectively. Applied voltages were 20 and $70 \mathrm{~V}$ for the ion transfer capillary and the tube lens, respectively. The ion transfer capillary was held at $275{ }^{\circ} \mathrm{C}$. Detection was achieved in the Orbitrap with a resolution set to 60,000 (at $\mathrm{m} / \mathrm{z} 400$ ) and a $\mathrm{m} / \mathrm{z}$ range between $110-1200$ in profile mode. Spectrum was analyzed using the acquisition software XCalibur 2.1 (Thermo Fisher Scientific, Courtaboeuf, France). The automatic gain control (AGC) allowed accumulation of up to 2.105 ions for FTMS scans, Maximum injection time was set to $300 \mathrm{~ms}$ and $1 \mu$ scan was acquired. $5 \mu \mathrm{L}$ was injected using a Thermo Finnigan Surveyor HPLC system (Thermo Fisher Scientific, Courtaboeuf, France) with a continuous infusion of methanol at $100 \mu \mathrm{L} / \mathrm{min}$.

Purification of solvents and reagents

All reagents and solvents were obtained from either Sigma-Aldrich, Acros, Alfa, VWR, or TCI and were used as received unless otherwise stated. Dry solvents were obtained from commercial sources. Grignard reagents were titrated before use according to a modified version of the Watson protocole. ${ }^{1}$ All reactions were conducted in dry flasks under argon atmosphere. Room temperature is ca. $22{ }^{\circ} \mathrm{C}$. 


\section{Synthesis of starting materials}

(1,Z)-bromohexene (1a) was prepared according to the previously reported literature procedures. ${ }^{2}$ NMR spectra were in agreement with reported data. ${ }^{3}$

${ }^{1} \mathrm{H}-\mathrm{NMR}$ (CDCl $\left.\mathbf{C}_{3}, 400 \mathrm{MHz}\right): 6.16$ - 6.07 (m, 2H), $2.24-2.18$ (m, 2H), $1.46-1.31$ (m, 4H), 0.93 (t, $J=7.2 \mathrm{~Hz}, 3 \mathrm{H})$.

${ }^{13} \mathbf{C}-\mathrm{NMR}\left(\mathbf{C D C l}_{\mathbf{3}}, \mathbf{7 5} \mathbf{M H z}\right):$ 135.2, 107.7, 30.4, 29.6, 22.4, 14.0.

2-butyl-1-bromohex-1-ene (1d) was prepared according to the previously reported literature procedures. ${ }^{4} \mathrm{NMR}$ spectra were in agreement with reported data. ${ }^{5}$

${ }^{1}$ H-NMR (CDCl 3 , 400 MHz): 5.87 (s, 1H), 2.23 - 2.19 (m, 2H), 2.12 - 2.08 (m, 2H), 1.46 - 1.26 (m, $8 \mathrm{H}), 0.92(\mathrm{dt}, J=10.8,7.1 \mathrm{~Hz}, 6 \mathrm{H})$.

${ }^{13} \mathbf{C}-\mathbf{N M R}\left(\mathbf{C D C l}_{3}\right.$, 75 MHz): 146.1, 100.9, 35.8, 32.5, 30.0, 29.4, 22.8, 22.5, 14.1, 14.0.

2-butyl-1-chlorohex-1-ene (1e) was prepared according to the previously reported literature procedures. ${ }^{4} \mathrm{NMR}$ spectra were in agreement with reported data.

${ }^{1} \mathbf{H}-N M R\left(\mathbf{C D C l}_{3}, 400 \mathrm{MHz}\right): 5.77(\mathrm{~s}, 1 \mathrm{H}), 2.19(\mathrm{t}, J=8.0 \mathrm{~Hz}, 2 \mathrm{H}), 2.04(\mathrm{t}, J=8.0 \mathrm{~Hz}, 2 \mathrm{H}), 1.44-$ $1.24(\mathrm{~m}, 8 \mathrm{H}), 0.91(\mathrm{dt}, J=9.0,7.1 \mathrm{~Hz}, 6 \mathrm{H})$.

${ }^{13} \mathbf{C}-\mathrm{NMR}\left(\mathbf{C D C l}_{3}\right.$, 75 MHz): 143.2, 111.8, 34.7, 30.0, 29.4, 22.8, 22.5, 14.1, 14.0.

Ethyl 4-(trifluoromethylsulfonate)-benzoate (3aa) was prepared according to the previously reported literature procedures. ${ }^{6} \mathrm{NMR}$ spectra were in agreement with reported data. ${ }^{7}$

${ }^{1} \mathbf{H}-\mathrm{NMR}\left(\mathbf{C D C l}_{\mathbf{3}}, \mathbf{4 0 0} \mathbf{M H z}\right): 8.18-8.14(\mathrm{~m}, 2 \mathrm{H}), 7.38-7.34(\mathrm{~m}, 2 \mathrm{H}), 4.41(\mathrm{q}, J=7.1 \mathrm{~Hz}, 2 \mathrm{H}), 1.41$ (t, $J=7.1 \mathrm{~Hz}, 3 \mathrm{H})$.

${ }^{13}$ C-NMR (CDCl 3 , 75 MHz): 165.1, 152.6, 132.0, 130.9, 121.5, 118.8 (q, $J=320.6 \mathrm{~Hz}$ ), 61.7, 14.4.

${ }^{19} \mathrm{~F}-\mathrm{NMR}\left(\mathrm{CDCl}_{3}, 282 \mathrm{MHz}\right):-72.73$ (s, 3F).

Ethyl 4-(phenylsulfonate)-benzoate (3ab) was prepared according to the previously reported literature procedures. ${ }^{8} \mathrm{NMR}$ spectra were in agreement with reported data.

${ }^{1} \mathbf{H}-\mathrm{NMR}\left(\mathbf{C D C l}_{\mathbf{3}}, \mathbf{4 0 0} \mathbf{M H z}\right): 8.01$ - $7.97(\mathrm{~m}, 2 \mathrm{H}), 7.85-7.83(\mathrm{~m}, 2 \mathrm{H}), 7.71-7.67$ (m, 1H), 7.56 $7.52(\mathrm{~m}, 2 \mathrm{H}), 7.08-7.04(\mathrm{~m}, 2 \mathrm{H}), 4.36(\mathrm{q}, J=7.1 \mathrm{~Hz}, 2 \mathrm{H}), 1.38(\mathrm{t}, J=7.1 \mathrm{~Hz}, 3 \mathrm{H})$.

${ }^{13} \mathbf{C}-\mathrm{NMR}$ (CDCl $\left.3 \mathbf{3}, \mathbf{7 5} \mathbf{M H z}\right): 165.6,152.9,135.2,134.6,131.4,129.5,129.4,128.6,122.4,61.4,14.4$.

Menthyl 4-chlorobenzoate (3c) was prepared according to the previously reported literature procedures. ${ }^{9} \mathrm{NMR}$ spectra were in agreement with reported data. ${ }^{9}$

${ }^{1}$ H-NMR (CDCl 3 , 400 MHz): $8.00-7.97$ (m, 2H), $7.43-7.40(\mathrm{~m}, 2 \mathrm{H}), 4.93$ (td, $J=10.9,4.4 \mathrm{~Hz}$, $1 \mathrm{H}), 2.15-2.09(\mathrm{~m}, 1 \mathrm{H}), 1.93(\mathrm{~m}, 1 \mathrm{H}), 1.77-1.71(\mathrm{~m}, 2 \mathrm{H}), 1.61-1.51(\mathrm{~m}, 2 \mathrm{H}), 1.19-1.06(\mathrm{~m}, 2 \mathrm{H})$, $0.99-0.88(\mathrm{~m}, 7 \mathrm{H}), 0.80(\mathrm{~d}, J=7.0 \mathrm{~Hz}, 3 \mathrm{H})$.

${ }^{13} \mathbf{C}-\mathbf{N M R}\left(\mathbf{C D C l}_{3}\right.$, $\left.7 \mathbf{5} \mathbf{M H z}\right): 165.4,139.3,131.1,129.4,128.8,75.3,47.4,41.1,34.4,31.6,26.7$, 23.8, 22.2, 20.9, 16.7 . 
Table S1: Optimization of the substrate concentration for alkyl-vinyl cross-coupling reaction

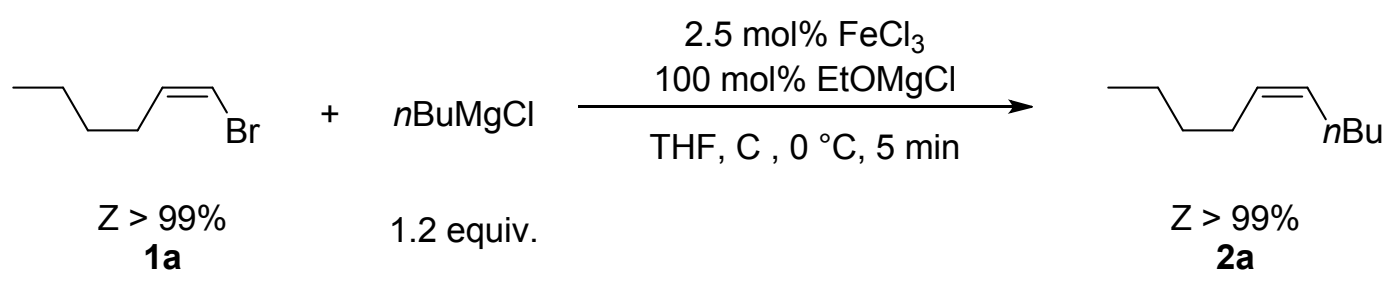

\begin{tabular}{ccc}
\hline Entry & Concentration $(\mathrm{mol} / \mathrm{L})$ & GC Yield (\%) \\
\hline 1 & 0.50 & 93 \\
2 & 0.20 & 77 \\
3 & 0.70 & 84 \\
\hline
\end{tabular}

Table S2: Optimization of the temperature for alkyl-vinyl cross-coupling reaction

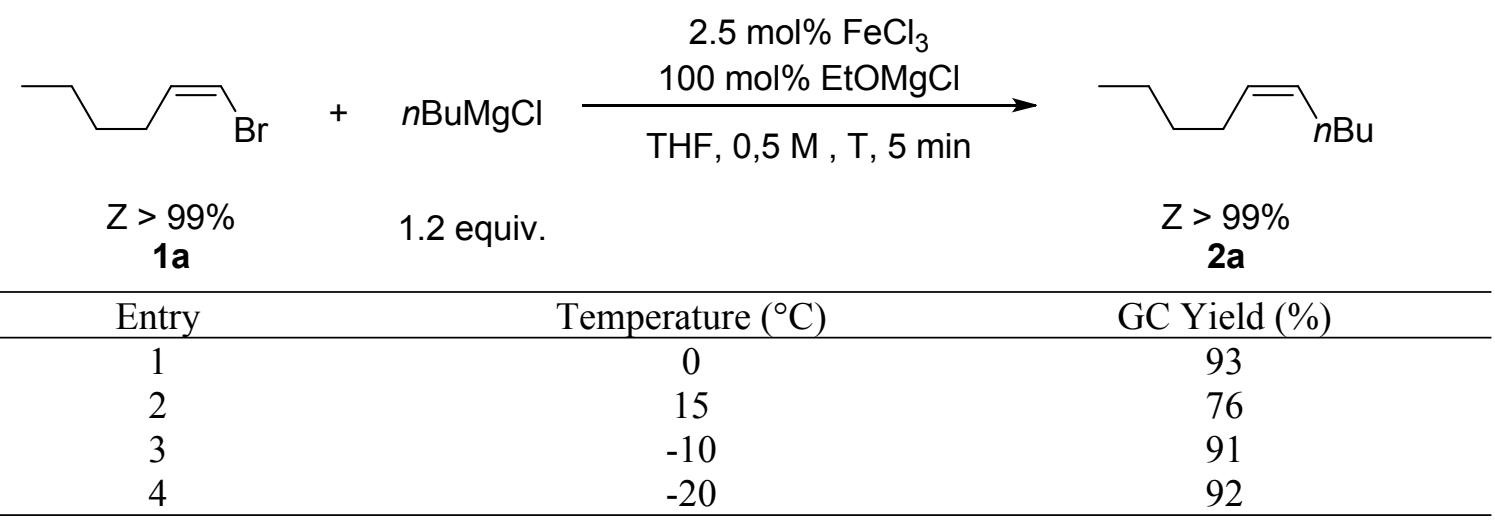

Table S3: Optimization of the nature of the RO[M] additive for alkyl-aryl crosscoupling reaction<smiles>CCOC(=O)c1ccc(Cl)cc1</smiles><smiles>CCCCc1ccc(C(=O)OCC)cc1</smiles>

3a

$4 a$

\begin{tabular}{ccc}
\hline Entry & Alcoolate & GC Yield (\%) \\
\hline 1 & EtOMgCl & 96 \\
2 & $n \mathrm{BuOMgCl}$ & 92 \\
3 & $\mathrm{MeOMgCl}$ & 93 \\
4 & $i \mathrm{PrOMgCl}$ & 78 \\
5 & $t \mathrm{BuOMgCl}_{2}$ & 84 \\
$6^{\mathrm{a}}$ & $\mathrm{ClMgOCH}_{2} \mathrm{CH}_{2} \mathrm{OMgCl}$ & 88 \\
7 & $\mathrm{PhOMgCl}^{2}$ & 74 \\
8 & $\mathrm{CF}_{3} \mathrm{CH}_{2} \mathrm{OMgCl}$ & 42 \\
9 & $\mathrm{AcOMgCl}$ & 72 \\
10 & $\mathrm{EtOLi}^{\mathrm{b}}$ & 27 \\
11 & $\mathrm{EtONa}^{\mathrm{c}}$ & 70 \\
12 & EtOK $^{\mathrm{c}}$ & 35
\end{tabular}


${ }^{*} 50 \mathrm{~mol} \%$ of $\mathrm{ClMgOCH}_{2} \mathrm{CH}_{2} \mathrm{OMgCl} ;{ }^{\mathrm{b}}$ prepared by metalation of EtOH with $\mathrm{nBuLi} ;{ }^{\mathrm{c}}$ commercial salt.

Table S4: Optimization of the quantity of $\mathrm{FeCl}_{3}$ for alkyl-aryl cross-coupling reaction<smiles>CCOC(=O)c1ccc(Cl)cc1</smiles>

3a

\begin{tabular}{ccc}
\hline Entry & Quantity of $\mathrm{FeCl}_{3}(\mathrm{x} \mathrm{mol} \%)$ & GC Yield (\%) \\
\hline 1 & 5 & 96 \\
2 & 2.5 & 94 \\
3 & 1 & 100 \\
4 & 0.5 & 87 \\
5 & 0.1 & 15 \\
\hline
\end{tabular}

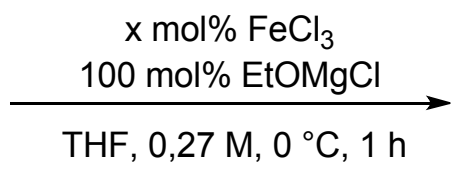<smiles>CCCCc1ccc(C(=O)OCC)cc1</smiles>

Table S5: Optimization of the quantity of EtOMgCl for alkyl-aryl cross-coupling reaction<smiles>CCOC(=O)c1ccc(Cl)cc1</smiles>

3a

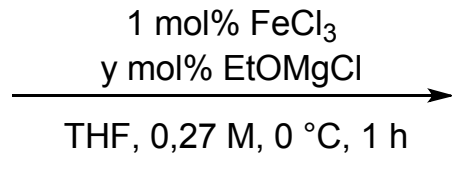

1.2 equiv

Entry

Quantity of EtOMgCl (y mol\%)

100

150

75

50

22

15

10<smiles>CCCCc1ccc(C(=O)OCC)cc1</smiles>

$4 a$

GC Yield $(\%)$
100
100
97
99
100
100
70


Table S6: Optimization of the temperature for alkyl-aryl cross-coupling reaction

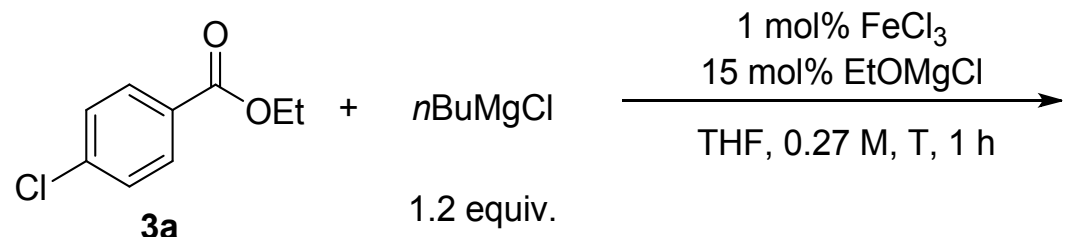<smiles>CCCCc1ccc(C(=O)OCC)cc1</smiles>

$\begin{array}{ccc}\text { Entry } & \text { Temperature }\left({ }^{\circ} \mathrm{C}\right) & \text { GC Yield }(\%) \\ 1 & 0 & 100 \\ 2 & 20 & 38 \\ 3 & 10 & 100 \\ 4 & -10 & 100 \\ 5 & -20 & 24\end{array}$

Table S7: Optimization of the substrate concentration for alkyl-aryl cross-coupling reaction

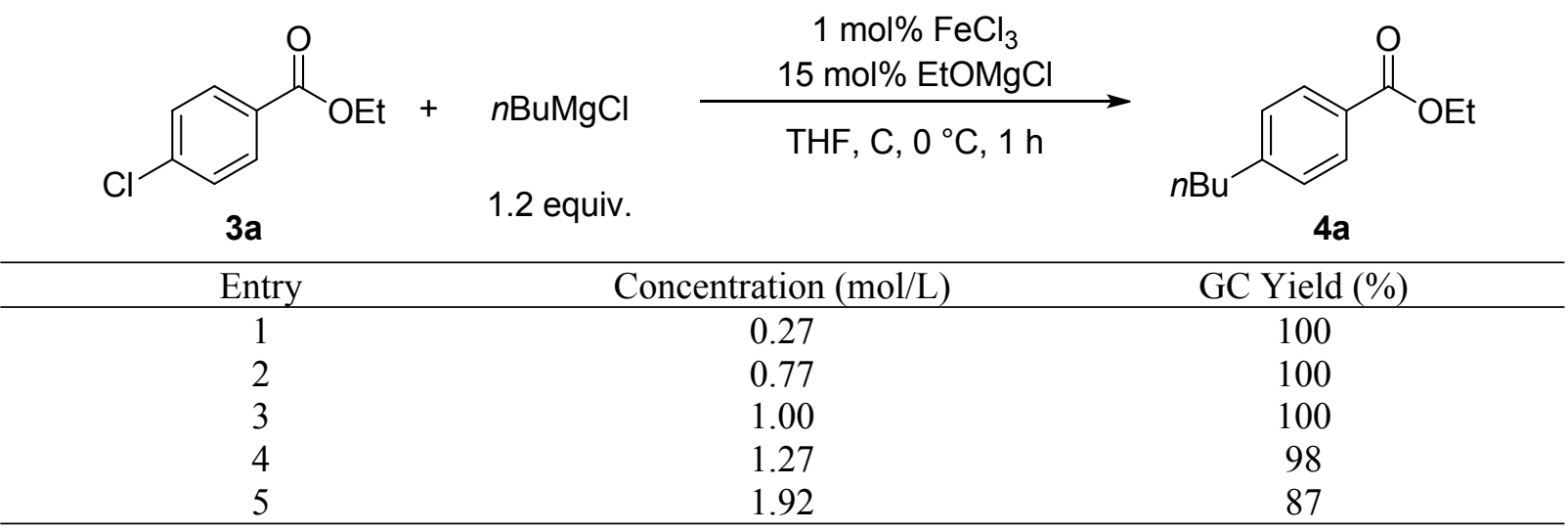




\section{General procedures for cross-coupling reactions}

General procedure for alkyl-vinyl cross-coupling reaction: In a dry $250 \mathrm{~mL}$ four-necked round bottom flask under argon with mechanical stirring were added EtOH (50 mmol, 1.00 equiv.) and THF (22.5 $\mathrm{mL}$ ). The solution was cooled to $0^{\circ} \mathrm{C}$ and isopropylmagnesium chloride ( $45.5 \mathrm{mmol}, 0.95$ equiv.) was added dropwise in $30 \mathrm{~min}$ with a syringe pump. The reaction mixture was stirred for $30 \mathrm{~min}$ at room temperature. A solution of $\mathrm{FeCl}_{3}$ in THF at $0.1 \mathrm{~mol} / \mathrm{L}$ ( $1.25 \mathrm{mmol}, 0.025$ equiv.) and the vinyl halide (50 mmol, 1.00 equiv.) were added. The solution was cooled to $0{ }^{\circ} \mathrm{C}$ and Grignard reagent $(65 \mathrm{mmol}$, 1.30 equiv.) was added dropwise in $30 \mathrm{~min}$ with a syringe pump. The reaction mixture was stirred for another $5 \mathrm{~min}$, quenched with an aqueous solution of $\mathrm{HCl} 1 \mathrm{~mol} / \mathrm{L}(130 \mathrm{~mL})$, extracted with diethyl ether $(3 \times 100 \mathrm{~mL})$ and the combined organic phase was carefully evaporated. The crude product was purified by distillation to obtain the product.

General procedure for alkyl-aryl cross-coupling reaction: In a dry $100 \mathrm{~mL}$ four-necked round bottom flask under argon with mechanical stirring were added EtOH (1.5 mmol, 1.00 equiv.) and THF (2 $\mathrm{mL}$ ). The solution was cooled to $0{ }^{\circ} \mathrm{C}$ and butylmagnesium chloride (1.65 mmol, 0.165 equiv.) was added dropwise in $1 \mathrm{~min}$ with a syringe pump. The reaction mixture was stirred for $30 \mathrm{~min}$ at room temperature. A solution of $\mathrm{FeCl}_{3}$ in THF at $0.1 \mathrm{~mol} / \mathrm{L}(0.1 \mathrm{mmol}, 0.01$ equiv.) and the aryl halide (10 mmol, 1.00 equiv.) were added. The solution was cooled to $0{ }^{\circ} \mathrm{C}$ and butylmagnesium chloride (12 mmol, 1.20 equiv.) was added dropwise in $5 \mathrm{~min}$ with a syringe pump. The reaction mixture was stirred for another $60 \mathrm{~min}$, quenched with an aqueous solution of $\mathrm{HCl} 1 \mathrm{~mol} / \mathrm{L}(15 \mathrm{~mL})$, extracted with diethyl ether $(3 \times 20 \mathrm{~mL})$ and the combined organic phase was carefully evaporated. The crude product was purified by column chromatography to obtain the product.

General procedure for alkyl-heteroaryl cross-coupling reaction: In a dry $100 \mathrm{~mL}$ four-necked round bottom flask under argon with mechanical stirring were added EtOH (1.5 mmol, 1.00 equiv. $)$ and THF ( $2 \mathrm{~mL}$ ). The solution was cooled to $0{ }^{\circ} \mathrm{C}$ and butylmagnesium chloride ( $1.65 \mathrm{mmol}, 0.165$ equiv.) was added dropwise in $1 \mathrm{~min}$ with a syringe pump. The reaction mixture was stirred for $30 \mathrm{~min}$ at room temperature. A solution of $\mathrm{FeCl}_{3}$ in THF at $0.1 \mathrm{~mol} / \mathrm{L}(0.1 \mathrm{mmol}, 0.01$ equiv. $)$ and the heteroaryl halide (10 mmol, 1.00 equiv.) were added. The solution was cooled to $0{ }^{\circ} \mathrm{C}$ and butylmagnesium chloride (12 mmol, 1.20 equiv.) was added dropwise in $5 \mathrm{~min}$ with a syringe pump. The reaction mixture was stirred for another $60 \mathrm{~min}$, quenched with water $(15 \mathrm{~mL})$, extracted with diethyl ether $(3 \times$ $20 \mathrm{~mL}$ ) and the combined organic phase was carefully evaporated. The crude product was purified by column chromatography to obtain the product.

General procedure for the one-pot amidification and alkyl-aryl cross-coupling reaction: In a dry 100 $\mathrm{mL}$ four-necked round bottom flask under argon with mechanical stirring were added secondary amine (11 mmol, 1.10 equiv.) and THF $(20 \mathrm{~mL})$. The solution was cooled to $0{ }^{\circ} \mathrm{C}$ and butylmagnesium chloride (12 mmol, 1.20 equiv.) was added dropwise in $5 \mathrm{~min}$ with a syringe pump. The reaction mixture was stirred for $30 \mathrm{~min}$ at room temperature followed by the addition of ethyl chlorobenzoate (10 mmol, 1.00 equiv.). The reaction mixture was stirred for another $30 \mathrm{~min}$ and a solution of $\mathrm{FeCl}_{3}$ in THF at $0.1 \mathrm{~mol} / \mathrm{L}\left(0.1 \mathrm{mmol}, 0.01\right.$ equiv.) was added. The solution was cooled to $0{ }^{\circ} \mathrm{C}$ and butylmagnesium chloride (12 mmol, 1.20 equiv.) was added dropwise in 5 min with a syringe pump. The reaction mixture was stirred for another $60 \mathrm{~min}$, quenched with an aqueous solution of $\mathrm{HCl} 1$ $\mathrm{mol} / \mathrm{L}(25 \mathrm{~mL})$, extracted with diethyl ether $(3 \times 20 \mathrm{~mL})$ and the combined organic phase was carefully evaporated. The crude product was purified by column chromatography to obtain the product. 


\section{Characterization Data}

All ${ }^{1} \mathrm{H}$ and ${ }^{13} \mathrm{C}$ NMR spectra of already reported compounds were in agreement with

literature. The corresponding references are cited thereafter.

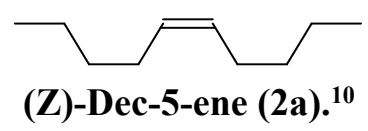

GC2010, Method 1: $t_{\mathrm{R}}=1.984 \mathrm{~min}$.

$\mathbf{T}_{\mathbf{e b}}=64{ }^{\circ} \mathrm{C} / 20$ Torr.

Purification: Distillation under reduced pressure.

Yield $=82 \%$ (5.75 g, colorless liquid).

${ }^{1}$ H-NMR (CDCl ${ }_{3}, 400$ MHz): 5.40 - 5.32 (m, 2H), 2.06 - 2.01 (m, 4H), 1.38 - 1.27 (m, 8H), 0.93 $0.89(\mathrm{~m}, 6 \mathrm{H})$.

${ }^{13} \mathrm{C}-\mathrm{NMR}\left(\mathrm{CDCl}_{3}, \mathbf{7 5} \mathbf{M H z}\right): 130.0,32.1,27.1,22.5,14.1$.

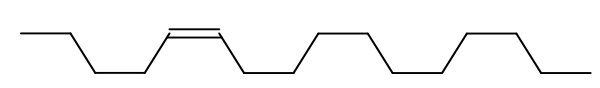

(Z)-Hexadec-5-ene (2b). ${ }^{11}$

GC2010, Method 1: $t_{\mathrm{R}}=4.816 \mathrm{~min}$.

$\mathbf{T}_{\mathrm{eb}}=105^{\circ} \mathrm{C} / 2$ Torr.

Purification: Distillation under reduced pressure.

Yield $=68 \%$ (7.61 g, colorless liquid).

${ }^{1}$ H-NMR (CDCl 3 , 400 MHz): 5.40 - 5.32 (m, 2H), 2.06 - 2.00 (m, 4H), 1.35 - 1.24 (m, 20H), 0.90 $(\mathrm{m}, 6 \mathrm{H})$.

${ }^{13} \mathbf{C}-\mathbf{N M R}\left(\mathbf{C D C l}_{3}, \mathbf{7 5} \mathbf{M H z}\right): 130.1,130.0,32.1,32.1,29.9,29.8,29.7,29.5,29.5,27.4,27.1,22.8$, $22.5,14.3,14.2$.

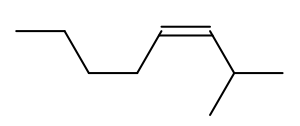

(Z)-2-Methyl-oct-3-ene (2c). ${ }^{12}$

GC2010, Method 1: $t_{\mathrm{R}}=0.959 \mathrm{~min}$.

$\mathbf{T}_{\mathrm{eb}}=115^{\circ} \mathrm{C}$.

Purification: Distillation under atmospheric pressure.

Yield $=65 \%(7.55 \mathrm{~g}$, colorless liquid $)$.

${ }^{1}$ H-NMR (CDCl ${ }_{3}, 400$ MHz): $5.28-5.16(\mathrm{~m}, 2 \mathrm{H}), 2.60(\mathrm{qd}, J=13.4,6.7 \mathrm{~Hz}, 1 \mathrm{H}), 2.04(\mathrm{q}, J=6.7$ $\mathrm{Hz}, 2 \mathrm{H}), 1.35-1.28(\mathrm{~m}, 4 \mathrm{H}), 0.96(\mathrm{~s}, 3 \mathrm{H}), 0.94(\mathrm{~s}, 3 \mathrm{H}), 0.92-0.89(\mathrm{~m}, 3 \mathrm{H})$.

${ }^{13} \mathbf{C}-\mathrm{NMR}\left(\mathbf{C D C l}_{3}\right.$, 75 MHz): 137.6, 127.6, 32.3, 27.2, 26.6, 23.4, 22.5, 14.1.

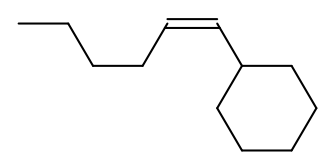

(Z)-1-Cyclohexanyl-hex-1-ene (2d). ${ }^{13}$

GC2010, Method 1: $t_{\mathrm{R}}=3.172 \mathrm{~min}$. 
$\mathbf{T}_{\mathbf{e b}}=75^{\circ} \mathrm{C} / 10$ Torr.

Purification: Distillation under reduced pressure.

Yield $=81 \%(6.72 \mathrm{~g}$, colorless liquid $)$.

${ }^{1} \mathbf{H}-\mathbf{N M R}\left(\mathbf{C D C l}_{\mathbf{3}}, \mathbf{4 0 0} \mathbf{M H z}\right): 5.29-5.17(\mathrm{~m}, 2 \mathrm{H}), 2.32-2.21(\mathrm{~m}, 1 \mathrm{H}), 2.04$ (q, $\left.J=6.8 \mathrm{~Hz}, 2 \mathrm{H}\right), 1.72$ $-1.62(\mathrm{~m}, 4 \mathrm{H}), 1.34-1.00(\mathrm{~m}, 10 \mathrm{H}), 0.91(\mathrm{t}, J=6.9 \mathrm{~Hz}, 3 \mathrm{H})$.

${ }^{13} \mathbf{C}-\mathbf{N M R}\left(\mathbf{C D C l}_{3}, \mathbf{7 5} \mathbf{M H z}\right): 136.2,128.2,36.4,33.6,32.4,27.3,26.2,26.2,22.5,14.2$.

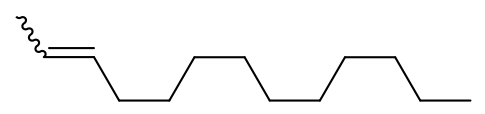

Tridec-2-ene (2e). ${ }^{14}$

GC2010, Method 1: $t_{\mathrm{R}}=3.613 \mathrm{~min}$.

$\mathbf{T}_{\mathbf{e b}}=113.7^{\circ} \mathrm{C} / 15.1$ Torr.

Purification: Distillation under reduced pressure.

Yield $=74 \%$ (6.76 g, colorless liquid).

${ }^{1} \mathrm{H}-\mathrm{NMR}\left(\mathrm{CDCl}_{3}, \mathbf{4 0 0} \mathbf{M H z}\right): 5.49-5.35(\mathrm{~m}, 2 \mathrm{H}), 2.07-1.94(\mathrm{~m}, 2 \mathrm{H}), 1.66-1.64(\mathrm{~m}, 1.7 \mathrm{H}), 1.62-$ $1.60(\mathrm{~m}, 1.3 \mathrm{H}), 1.35-1.27(\mathrm{~m}, 16 \mathrm{H}), 0.89(\mathrm{t}, J=6.8 \mathrm{~Hz}, 3 \mathrm{H})$.

${ }^{13} \mathbf{C}-\mathbf{N M R}\left(\mathbf{C D C l}_{3}, \mathbf{7 5} \mathbf{M H z}\right): 131.8,131.1,124.7,123.7,32.8,32.1,29.8,29.7,29.5,29.4,27.0,22.9$, 18.1, 14.3.

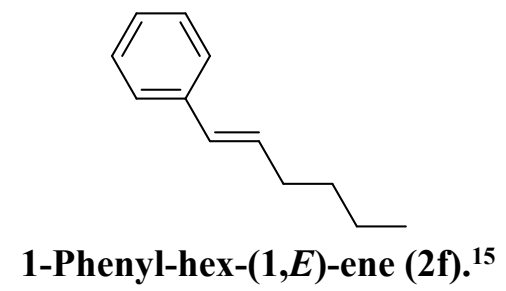

This synthesis has been carried out on $10 \mathrm{mmol}$ of starting material (1c).

GC2010, Method 1: $t_{\mathrm{R}}=3.774 \mathrm{~min}$.

TLC: $R_{f}=0.38$, Eluent: Petroleum spirit.

Purification: Chromatography, Eluent: Petroleum spirit.

Yield $=94 \%(1.50 \mathrm{~g}$, colorless liquid $)$.

${ }^{1}$ H-NMR (CDCl 3 , 400 MHz): $7.27-7.10(\mathrm{~m}, 5 \mathrm{H}), 6.38(\mathrm{~m}, 1 \mathrm{H}), 6.15(\mathrm{dt}, J=15.8,6.8 \mathrm{~Hz}, 1 \mathrm{H}), 2.32$ $-2.08(\mathrm{~m}, 2 \mathrm{H}), 1.41-1.25(\mathrm{~m}, 4 \mathrm{H}), 0.83(\mathrm{t}, J=7.2 \mathrm{~Hz}, 3 \mathrm{H})$.

${ }^{13} \mathbf{C}-\mathrm{NMR}$ (CDCl $\left.\mathbf{3}, \mathbf{7 5} \mathbf{M H z}\right):$ 138.1, 131.4, 129.8, 128.6, 126.9, 126.0, 32.9, 31.7, 22.4, 14.1.

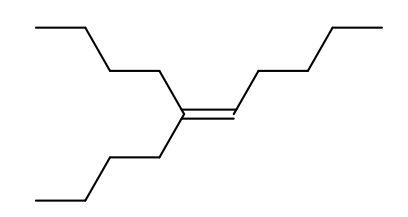

\section{5-Butyl-dec-5-ene (2g). ${ }^{13}$}

GC2010, Method 1: $t_{\mathrm{R}}=3.652 \mathrm{~min}$.

$\mathbf{T}_{\text {eb }}=99.5^{\circ} \mathrm{C} / 8.3$ Torr.

Purification: Distillation under reduced pressure.

Yield $_{\text {chloride }}=20 \%$ (1.99 g, colorless liquid), Yield bromide $=37 \%$ (3.64 g, colorless liquid).

${ }^{1} \mathrm{H}-\mathrm{NMR}\left(\mathbf{C D C l}_{3}, \mathbf{4 0 0} \mathbf{M H z}\right): 5.10(\mathrm{t}, J=7.0 \mathrm{~Hz}, 1 \mathrm{H}), 2.02-1.95(\mathrm{~m}, 6 \mathrm{H}), 1.41-1.24(\mathrm{~m}, 12 \mathrm{H})$, $0.91(\mathrm{~m}, 9 \mathrm{H})$.

${ }^{13} \mathbf{C}-\mathbf{N M R}\left(\mathbf{C D C l}_{\mathbf{3}}, \mathbf{7 5} \mathbf{M H z}\right):$ 139.7, 124.8, 36.8, 32.6, 30.9, 30.7, 29.9, 27.6, 23.0, 22.7, 22.6, 14.2. 


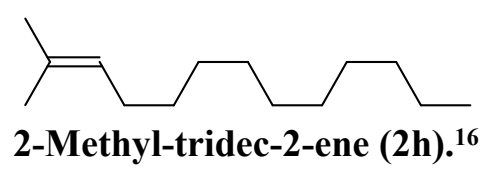

GC2010, Method 1: $t_{\mathrm{R}}=3.701 \mathrm{~min}$.

$\mathbf{T}_{\mathrm{eb}}=99.6^{\circ} \mathrm{C} / 4.5$ Torr.

Purification: Distillation under reduced pressure.

Yield $_{\text {chloride }}=31 \%$ (3.00 g, colorless liquid), Yield bromide $=66 \%(6.43 \mathrm{~g}$, colorless liquid $)$.

${ }^{1} \mathrm{H}-\mathrm{NMR}\left(\mathbf{C D C l}_{3}, \mathbf{4 0 0} \mathbf{M H z}\right): 5.14(\mathrm{t}, J=7.8 \mathrm{~Hz}, 1 \mathrm{H}), 1.98(\mathrm{~m}, 2 \mathrm{H}), 1.71$ (s, 3H), 1.62 (s, 3H), 1.29 (m, 16H), $0.90(\mathrm{t}, J=6.8 \mathrm{~Hz}, 3 \mathrm{H})$.

${ }^{13} \mathbf{C}-\mathbf{N M R}\left(\mathbf{C D C l}_{3}, \mathbf{7 5} \mathbf{M H z}\right): 131.2,125.2,32.1,30.1,29.9,29.8,29.8,29.5,28.2,25.9,22.9,17.8$, 14.3.

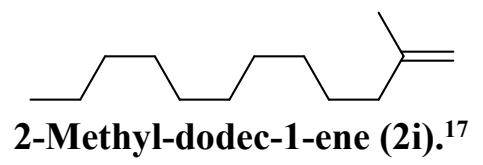

GC2010, Method 1: $t_{\mathrm{R}}=3.525 \mathrm{~min}$.

$\mathbf{T}_{\mathbf{e b}}=85.7^{\circ} \mathrm{C} / 5.3$ Torr.

Purification: Distillation under reduced pressure.

Yield $=81 \%(7.38 \mathrm{~g}$, colorless liquid $)$.

${ }^{1} \mathrm{H}-\mathrm{NMR}\left(\mathbf{C D C l}_{3}, 400 \mathrm{MHz}\right): 4.68(\mathrm{~d}, J=8.8 \mathrm{~Hz}, 2 \mathrm{H}), 2.16-1.90(\mathrm{t}, J=2.0 \mathrm{~Hz}, 2 \mathrm{H}), 1.72(\mathrm{~s}, 3 \mathrm{H})$, $1.47-1.37$ (m, 2H), $1.28(\mathrm{~m}, 16 \mathrm{H}), 0.89$ (t, $J=6.8 \mathrm{~Hz}, 3 \mathrm{H})$.

${ }^{13} \mathbf{C}-\mathrm{NMR}\left(\mathbf{C D C l}_{3}\right.$, 75 MHz): 146.3, 109.4, 37.8, 31.8, 29.5, 29.4, 29.2, 27.5, 22.6, 22.3, 14.0.

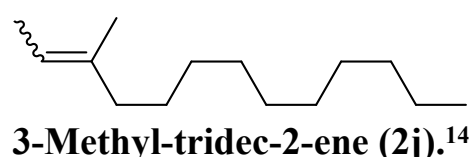

GC2010, Method 1: $t_{\mathrm{R}}=4.024 \mathrm{~min}$.

$\mathbf{T}_{\mathrm{eb}}=112^{\circ} \mathrm{C} / 8$ Torr.

Purification: Distillation under reduced pressure.

Yield $=63 \%$ (6.21 g, colorless liquid).

${ }^{1} \mathrm{H}-\mathrm{NMR}\left(\mathbf{C D C l}_{3}, \mathbf{4 0 0} \mathbf{M H z}\right): 5.24-5.18(\mathrm{~m}, 1 \mathrm{H}), 2.00(\mathrm{dt}, J=21.8,7.5 \mathrm{~Hz}, 2 \mathrm{H}), 1.70-1.57$ (m, $6 \mathrm{H}), 1.43-1.28(\mathrm{~m}, 16 \mathrm{H}), 0.91(\mathrm{t}, J=6.8 \mathrm{~Hz}, 3 \mathrm{H})$.

${ }^{13} \mathbf{C}-\mathrm{NMR}\left(\mathbf{C D C l}_{3}, \mathbf{7 5} \mathbf{M H z}\right): 136.6,136.4,118.8,118.1,39.9,32.1,31.5,29.8,29.8,29.5,28.2,28.0$, 23.6, 22.9, 15.7, 14.3, 13.5, 13.4 .

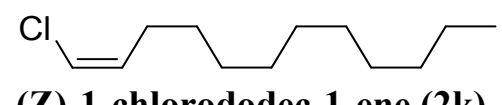

GC2010, Method 1: $t_{\mathrm{R}}=4.155 \mathrm{~min}$.

$\mathbf{T}_{\mathrm{eb}}=117.0^{\circ} \mathrm{C} / 9.8$ Torr.

Purification: Distillation under reduced pressure.

Yield $=31 \%(3.14 \mathrm{~g}$, colorless liquid $)$.

${ }^{1} \mathrm{H}-\mathrm{NMR}\left(\mathbf{C D C l}_{3}, \mathbf{4 0 0} \mathbf{M H z}\right): 6.01(\mathrm{dt}, J=7.0,1.5 \mathrm{~Hz}, 1 \mathrm{H}), 5.76(\mathrm{q}, J=7.1 \mathrm{~Hz}, 1 \mathrm{H}), 2.23$ (qd, $J=$ $7.3,1.5 \mathrm{~Hz}, 2 \mathrm{H}), 1.31-1.27$ (m, 16H), 0.89 (t, $J=6.8 \mathrm{~Hz}, 3 \mathrm{H})$.

${ }^{13} \mathbf{C}-\mathrm{NMR}\left(\mathbf{C D C l}_{\mathbf{3}}, \mathbf{7 5} \mathbf{M H z}\right): 132.1,117.9,32.1,29.7,29.6,29.5,29.3,28.5,27.2,22.8,14.3$.

MS (EI, $70 \mathrm{eV}) \mathbf{~ m / z}: 204\left(\mathrm{M}^{+\bullet}, 33\right), 202\left(\mathrm{M}^{+\bullet}, 100\right)$. 


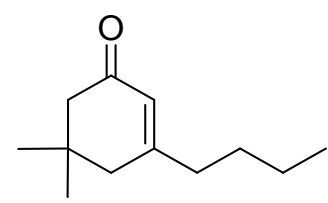

3-butyl-5,5-dimethylcyclohex-2-en-1-one (2l). ${ }^{13}$

This synthesis has been carried out on $10 \mathrm{mmol}$ of starting material (1k).

TLC: $R_{f}=0.79$, Eluent: Petroleum spirit/Ethyl acetate 80:20.

Purification: Chromatography, Eluent: Petroleum spirit/Ethyl acetate 80:20.

Yield $=87 \%(1.56 \mathrm{~g}$, colorless liquid).

${ }^{1} \mathrm{H}-\mathrm{NMR}\left(\mathbf{C D C l}_{\mathbf{3}}, \mathbf{4 0 0} \mathbf{M H z}\right): 5.85(\mathrm{~s}, 1 \mathrm{H}), 2.17(\mathrm{~d}, J=14.5 \mathrm{~Hz}, 6 \mathrm{H}), 1.50-1.42(\mathrm{~m}, 2 \mathrm{H}), 1.37-1.27$ (m, 2H), $1.01(\mathrm{~s}, 6 \mathrm{H}), 0.90(\mathrm{t}, J=7.3 \mathrm{~Hz}, 3 \mathrm{H})$.

${ }^{13}$ C-NMR (CDCl $\mathbf{3}$, 75 MHz): 200.2, 164.4, 124.7, 51.2, 44.0, 37.8, 33.7, 29.0, 28.4, 22.4, 13.9.<smiles>CCCCc1ccc(C(=O)OCC)cc1</smiles>

Ethyl $p$-butylbenzoate (4a). ${ }^{15}$

GC2014, Method 2: $t_{\mathrm{R}}=3.822 \mathrm{~min}$.

Yield $=99 \%(2.06 \mathrm{~g}$, yellow oil $)$.

${ }^{1} \mathbf{H}-N M R\left(\mathbf{C D C l}_{3}, \mathbf{4 0 0} \mathbf{M H z}\right): 7.96(\mathrm{~d}, J=8.2 \mathrm{~Hz}, 2 \mathrm{H}), 7.25(\mathrm{~d}, J=8.1 \mathrm{~Hz}, 2 \mathrm{H}), 4.37(\mathrm{q}, J=7.1 \mathrm{~Hz}$, 2H), $2.69-2.64(\mathrm{~m}, 2 \mathrm{H}), 1.67-1.58(\mathrm{~m}, 2 \mathrm{H}), 1.37$ (dt, $J=15.1,7.2 \mathrm{~Hz}, 5 \mathrm{H}), 0.94(\mathrm{t}, J=7.3 \mathrm{~Hz}, 3 \mathrm{H})$.

${ }^{13} \mathbf{C}-\mathrm{NMR}$ (CDCl $\left.\mathbf{C D}_{3}, \mathbf{7 5} \mathbf{M H z}\right): 166.9,148.5,129.7,128.5,128.1,60.9,35.8,33.4,22.4,14.5,14.0$.

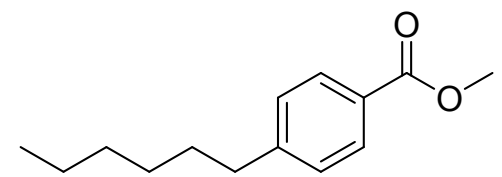

Methyl $p$-hexylbenzoate (4b). ${ }^{18}$

GC2014, Method 2: $t_{\mathrm{R}}=4.526 \mathrm{~min}$.

Yield $=99 \%$ (2.22 g, yellow oil).

Purification: No further purification was required.

${ }^{1} \mathrm{H}-\mathrm{NMR}\left(\mathbf{C D C l}_{3}, \mathbf{4 0 0} \mathbf{M H z}\right): \delta 7.97-7.94(\mathrm{~m}, 2 \mathrm{H}), 7.25(\mathrm{~d}, J=8.3 \mathrm{~Hz}, 2 \mathrm{H}), 3.91(\mathrm{~s}, 3 \mathrm{H}), 2.68-$ $2.64(\mathrm{~m}, 2 \mathrm{H}), 1.67-1.61(\mathrm{~m}, 2 \mathrm{H}), 1.36-1.28(\mathrm{~m}, 6 \mathrm{H}), 0.90-0.87(\mathrm{~m}, 3 \mathrm{H})$.

${ }^{13} \mathbf{C}-N M R\left(\mathbf{C D C l}_{3}, \mathbf{7 5} \mathbf{M H z}\right): 167.4,148.7,129.8,128.6,127.7,52.1,36.2,31.8,31.2,29.1,22.7$, 14.2 .

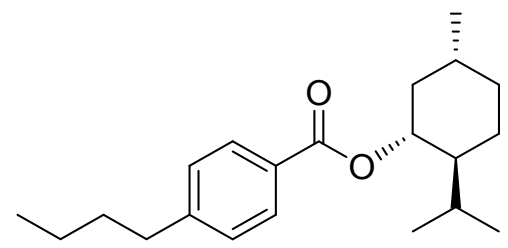

Menthyl $p$-butylbenzoate (4c).

GC2014, Method 2: $t_{\mathrm{R}}=8.019 \mathrm{~min}$.

Yield $=99 \%(3.30 \mathrm{~g}$, orange oil $)$.

Purification: No further purification was required.

${ }^{1} \mathbf{H}-\mathrm{NMR}\left(\mathbf{C D C l}_{3}, \mathbf{4 0 0} \mathbf{M H z}\right): \delta 7.97-7.95(\mathrm{~m}, 2 \mathrm{H}), 7.25(\mathrm{~d}, J=8.3 \mathrm{~Hz}, 2 \mathrm{H}), 4.93(\mathrm{td}, J=10.9,4.4$ $\mathrm{Hz}, 1 \mathrm{H}), 2.69-2.65(\mathrm{~m}, 2 \mathrm{H}), 2.15-2.10(\mathrm{~m}, 1 \mathrm{H}), 1.97$ (dtd, $J=13.9,7.0,2.7 \mathrm{~Hz}, 1 \mathrm{H}), 1.76-1.71$ 
$(\mathrm{m}, 2 \mathrm{H}), 1.66-1.51(\mathrm{~m}, 4 \mathrm{H}), 1.36(\mathrm{dq}, J=14.6,7.3 \mathrm{~Hz}, 2 \mathrm{H}), 1.19-1.05(\mathrm{~m}, 2 \mathrm{H}), 0.98-0.89(\mathrm{~m}$, $10 \mathrm{H}), 0.80(\mathrm{~d}, J=7.0 \mathrm{~Hz}, 3 \mathrm{H})$.

${ }^{13} \mathbf{C}-N M R\left(\mathbf{C D C l}_{3}, \mathbf{7 5} \mathbf{M H z}\right): 166.3,148.4,129.8,128.5,128.5,74.7,47.4,41.2,35.8,34.5,33.5$, $31.6,26.6,23.8,22.5,22.2,20.9,16.7,14.1$.

HRMS (ESI): [M + Na $]^{+}$Calculated for $\mathrm{C}_{21} \mathrm{H}_{32} \mathrm{O}_{2} \mathrm{Na}$ : 339.2295; Found: 339.2292.

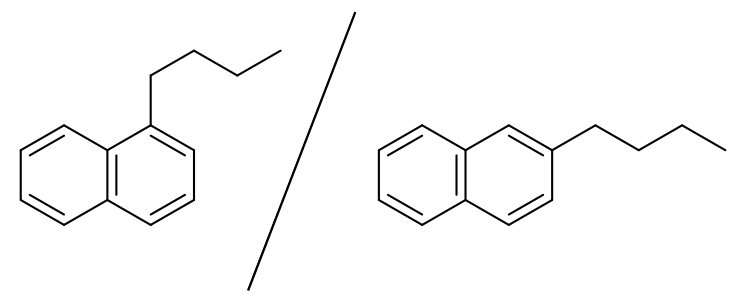

85:15

1-butylnaphthalene/2-butylnaphthalene 85:15 (4d). ${ }^{19}$

GC2014, Method 2: $t_{\mathrm{R}}=3.950 \mathrm{~min}$.

TLC: $R_{f}=0.54$, Eluent: Petroleum spirit.

Purification: Chromatography, Eluent: Petroleum spirit.

Yield $=85 \%$ (1.57 g, colorless liquid).

${ }^{1} \mathrm{H}-\mathrm{NMR}\left(\mathbf{C D C l}_{\mathbf{3}}, \mathbf{4 0 0} \mathbf{M H z}\right): 8.07$ (d, $\left.J=8.1 \mathrm{~Hz}, 0.85 \mathrm{H}\right), 7.87$ (d, $\left.J=7.7 \mathrm{~Hz}, 0.85 \mathrm{H}\right), 7.83-7.77$ (m, $0.45 \mathrm{H}), 7.72(\mathrm{~d}, J=8.1 \mathrm{~Hz}, 0.85 \mathrm{H}), 7.63(\mathrm{~s}, 0.15 \mathrm{H}), 7.55-7.46(\mathrm{~m}, 1.70 \mathrm{H}), 7.45-7.39(\mathrm{~m}, 1 \mathrm{H}), 7.36$ $-7.33(\mathrm{~m}, 1 \mathrm{H}), 7.27(\mathrm{~d}, J=2.1 \mathrm{~Hz}, 0.15 \mathrm{H}), 3.09(\mathrm{t}, J=8.0 \mathrm{~Hz}, 1.70 \mathrm{H}), 2.80(\mathrm{t}, J=7.7 \mathrm{~Hz}, 0.30 \mathrm{H})$, $1.80-1.69(\mathrm{~m}, 2 \mathrm{H}), 1.53-1.39(\mathrm{~m}, 2 \mathrm{H}), 0.99(\mathrm{t}, J=7.4 \mathrm{~Hz}, 3 \mathrm{H})$.

${ }^{13} \mathbf{C}-N M R\left(\mathbf{C D C l}_{3}, \mathbf{7 5} \mathbf{~ M H z}\right): 140.6,139.1,134.0,133.8,132.1,128.9,127.9,127.7,127.6,127.5$, $126.5,126.4,126.0,125.7,125.7,125.5,125.1,124.1,36.0,33.7,33.2,33.0,23.0,22.5,14.2$.

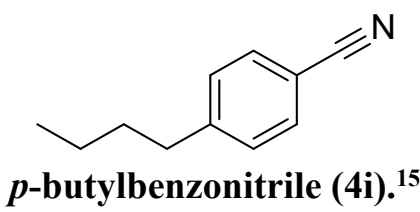

GC2014, Method 2: $t_{\mathrm{R}}=2.897 \mathrm{~min}$.

TLC: $R_{f}=0.21$, Eluant: Petroleum spirit/Ethyl acetate 95:5.

Purification: Chromatography, Eluant: Petroleum spirit/Ethyl acetate 95:5.

Yield $=80 \%(1.27 \mathrm{~g}$, colorless liquid $)$.

${ }^{1} \mathbf{H}-\mathbf{N M R}\left(\mathbf{C D C l}_{3}, \mathbf{4 0 0} \mathbf{M H z}\right): 7.58(\mathrm{~d}, J=8.2 \mathrm{~Hz}, 2 \mathrm{H}), 7.29(\mathrm{~d}, J=8.2 \mathrm{~Hz}, 2 \mathrm{H}), 2.72-2.66(\mathrm{~m}, 2 \mathrm{H})$, $1.66-1.58(\mathrm{~m}, 2 \mathrm{H}), 1.42-1.33(\mathrm{dq}, J=14.6,7.3 \mathrm{~Hz}, 2 \mathrm{H}), 0.95(\mathrm{t}, J=7.3 \mathrm{~Hz}, 3 \mathrm{H})$.

${ }^{13}$ C-NMR (CDCl 3 , 75 MHz): 148.7, 132.2, 129.3, 119.4, 109.6, 36.0, 33.2, 22.4, 14.0.

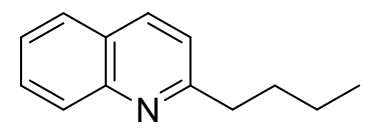

2-butylquinoline (4k). ${ }^{20}$

GC2014, Method 2: $t_{\mathrm{R}}=4.054 \mathrm{~min}$.

Yield $=97 \%(1.79 \mathrm{~g}$, yellow oil $)$.

Purification: No further purification was required.

${ }^{1} \mathrm{H}-\mathrm{NMR}\left(\mathbf{C D C l}_{3}, 400 \mathrm{MHz}\right): 8.09(\mathrm{~d}, J=8.2 \mathrm{~Hz}, 2 \mathrm{H}), 7.79(\mathrm{~d}, J=8.1 \mathrm{~Hz}, 1 \mathrm{H}), 7.70$ (t, $J=7.6 \mathrm{~Hz}$, $1 \mathrm{H}), 7.50(\mathrm{t}, J=7.5 \mathrm{~Hz}, 1 \mathrm{H}), 7.32(\mathrm{~d}, J=8.4 \mathrm{~Hz}, 1 \mathrm{H}), 3.00(\mathrm{t}, J=8.0 \mathrm{~Hz}, 2 \mathrm{H}), 1.85-1.77(\mathrm{~m}, 2 \mathrm{H})$, $1.51-1.41$ (sex, $J=8.0 \mathrm{~Hz}, 2 \mathrm{H}), 0.97$ (t, $J=7.4 \mathrm{~Hz}, 3 \mathrm{H})$. 
${ }^{13} \mathbf{C}-N M R\left(\mathbf{C D C l}_{3}, \mathbf{7 5}\right.$ MHz): 163.2, 147.9, 136.4, 129.5, 128.8, 127.6, 126.8, 125.8, 121.5, 39.2, 32.3, $22.8,14.1$.<smiles>CCCCc1ncccn1</smiles>

\section{2-butylpyrimidine (4I). ${ }^{21}$}

This synthesis has been carried out on $50 \mathrm{mmol}$ of starting material $(\mathbf{3 m})$.

GC2014, Method 2: $t_{\mathrm{R}}=1.221 \mathrm{~min}$.

Yield $=86 \%$ (5.82 g, colorless liquid).

Purification: No further purification was required.

${ }^{1} \mathrm{H}-\mathrm{NMR}\left(\mathbf{C D C l}_{\mathbf{3}}, \mathbf{4 0 0} \mathbf{M H z}\right): 8.68(\mathrm{~d}, J=4.9 \mathrm{~Hz}, 2 \mathrm{H}), 7.13(\mathrm{t}, J=4.9 \mathrm{~Hz}, 1 \mathrm{H}), 2.98(\mathrm{t}, J=8.0 \mathrm{~Hz}$, $2 \mathrm{H}), 1.86-1.78(\mathrm{~m}, 2 \mathrm{H}), 1.42(\mathrm{sex}, J=7.2 \mathrm{~Hz}, 2 \mathrm{H}), 0.96(\mathrm{t}, J=7.4 \mathrm{~Hz}, 3 \mathrm{H})$.

${ }^{13} \mathbf{C}-\mathbf{N M R}\left(\mathbf{C D C l}_{3}, \mathbf{7 5} \mathbf{M H z}\right): 171.8,157.1,118.5,39.4,31.0,22.7,14.1$.<smiles>CCCCc1ccc(C(=O)N(CC)CC)cc1</smiles>

$N, N$-diethyl $p$-butyl-benzamide (5a).

GC2014, Method 2: $t_{\mathrm{R}}=5.665 \mathrm{~min}$.

TLC: $R_{f}=0.13$, Eluent: Petroleum spirit/Ethyl acetate 90:10.

Purification: Chromatography, Eluent: Petroleum spirit/Ethyl acetate 90:10.

Yield $=96 \%(2.23 \mathrm{~g}$, yellow oil $)$.

${ }^{1} \mathbf{H}-\mathrm{NMR}\left(\mathbf{C D C l}_{\mathbf{3}}, \mathbf{4 0 0} \mathbf{M H z}\right): 7.29-7.27(\mathrm{~m}, 2 \mathrm{H}), 7.18(\mathrm{~d}, J=8.1 \mathrm{~Hz}, 2 \mathrm{H}), 3.40$ (d, $\left.J=76.3 \mathrm{~Hz}, 4 \mathrm{H}\right)$, $2.62(\mathrm{t}, J=6.0 \mathrm{~Hz}, 2 \mathrm{H}), 1.63-1.56(\mathrm{~m}, 2 \mathrm{H}), 1.35(\mathrm{sex}, J=7.2 \mathrm{~Hz}, 2 \mathrm{H}), 1.17$ (s, 6H), 0.92 (t, $J=7.3$ $\mathrm{Hz}, 3 \mathrm{H})$.

${ }^{13} \mathbf{C}-\mathbf{N M R}\left(\mathbf{C D C l}_{3}, \mathbf{7 5} \mathbf{M H z}\right): 171.6,144.1,134.6,128.4,126.4,43.4,39.3,35.5,33.5,22.3,14.3$, 14.0, 13.0.

HRMS (ESI): $[\mathrm{M}+\mathrm{Na}]^{+}$Calculated for $\mathrm{C}_{15} \mathrm{H}_{23} \mathrm{NONa}$ : 256.1672; Found: 256.1662 .

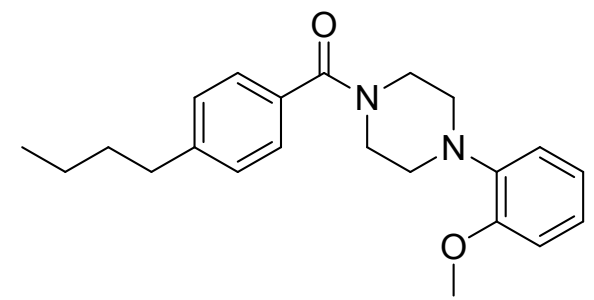

(4-butylphenyl)-(o-anisylpiperazinyl)methanone (5b).

GC2014, Method 3: $t_{\mathrm{R}}=16.595 \mathrm{~min}$.

TLC: $R_{f}=0.10$, Eluent: Petroleum spirit/Diethyl ether 50:50.

Purification: Chromatography, Eluent: Petroleum spirit/Diethyl ether 50:50.

Yield $=90 \%$ (3.17 g, white solid).

${ }^{1} \mathrm{H}-\mathrm{NMR}\left(\mathbf{C D C l}_{3}, \mathbf{4 0 0} \mathbf{M H z}\right): 7.28(\mathrm{~d}, J=8.0 \mathrm{~Hz}, 2 \mathrm{H}), 7.14(\mathrm{~d}, J=8.0 \mathrm{~Hz}, 2 \mathrm{H}), 6.99-6.95(\mathrm{~m}, 1 \mathrm{H})$, $6.86-6.80(\mathrm{~m}, 3 \mathrm{H}), 3.80(\mathrm{~s}, 3 \mathrm{H}), 3.73(\mathrm{~d}, J=127.8 \mathrm{~Hz}, 4 \mathrm{H}), 3.00(\mathrm{~d}, J=41.9 \mathrm{~Hz}, 4 \mathrm{H}), 2.56(\mathrm{t}, J=8.0$ $\mathrm{Hz}, 2 \mathrm{H}), 1.57-1.49$ (m, 2H), $1.28(\mathrm{sex}, J=7.2 \mathrm{~Hz}, 2 \mathrm{H}), 0.85$ (t, $J=7.3 \mathrm{~Hz}, 3 \mathrm{H})$.

${ }^{13} \mathbf{C}-N M R\left(\mathbf{C D C l}_{3}, \mathbf{7 5} \mathbf{~ M H z}\right): 170.7,152.4,145.0,140.8,133.1,128.6,127.3,123.7,121.2,118.6$, 111.4, 55.5, 51.3, 50.9, 48.2, 42.5, 35.6, 33.6, 22.4, 14.0.

HRMS (ESI): [M + Na] $]^{+}$Calculated for $\mathrm{C}_{22} \mathrm{H}_{28} \mathrm{~N}_{2} \mathrm{O}_{2} \mathrm{Na}$ : 375.2043; Found: 375.2044 . 
<smiles>CCCCc1ccc(C(=O)N2CCOCC2)cc1</smiles>

(4-butylphenyl)-4-morpholinylmethanone (5c). ${ }^{22}$

GC2014, Method 2: $t_{\mathrm{R}}=7.607 \mathrm{~min}$.

Yield $=99 \%$ (2.48 g, orange oil).

Purification: No further purification was required.

${ }^{1} \mathrm{H}-\mathrm{NMR}\left(\mathbf{C D C l}_{3}, \mathbf{4 0 0} \mathbf{M H z}\right): 7.41-7.21(\mathrm{~m}, 4 \mathrm{H}), 3.97-3.33$ (m, 8H), 2.63 (t, $\left.J=8.0 \mathrm{~Hz} 2 \mathrm{H}\right), 1.59$ (quint, $J=7.0 \mathrm{~Hz}, 2 \mathrm{H}), 1.35$ (sex, $J=7.2 \mathrm{~Hz}, 2 \mathrm{H}), 0.92(\mathrm{t}, J=7.3 \mathrm{~Hz}, 3 \mathrm{H})$.

${ }^{13} \mathbf{C}-\mathbf{N M R}\left(\mathbf{C D C l}_{3}, \mathbf{7 5} \mathbf{M H z}\right): 170.7,145.1,132.6,128.6,127.3,67.0,48.4,42.9,35.6,33.5,22.3$, 14.0.<smiles>CCCCc1ccc(C(=O)N(C)c2ccccc2)cc1</smiles>

$N$-methyl- $N$-phenyl $p$-butyl-benzamide (5d).

GC2014, Method 2: $t_{\mathrm{R}}=8.155 \mathrm{~min}$.

TLC: $R_{f}=0.60$, Eluent: Petroleum spirit/Diethyl ether 50:50.

Purification: Chromatography, Eluent: Petroleum spirit/Diethyl ether 50:50.

Yield $=99 \%$ (2.64 g, orange oil).

${ }^{1} \mathrm{H}-\mathrm{NMR}\left(\mathbf{C D C l}_{\mathbf{3}}, \mathbf{4 0 0} \mathbf{M H z}\right): 7.25$ - $7.20(\mathrm{~m}, 4 \mathrm{H}), 7.16$ - $7.12(\mathrm{~m}, 1 \mathrm{H}), 7.05-7.03(\mathrm{~m}, 2 \mathrm{H}), 6.97$ (d, $J=8.1 \mathrm{~Hz}, 2 \mathrm{H}), 3.50(\mathrm{~s}, 3 \mathrm{H}), 2.53-2.49(\mathrm{~m}, 2 \mathrm{H}), 1.55-1.47(\mathrm{~m}, 2 \mathrm{H}), 1.33-1.22(\mathrm{~m}, 2 \mathrm{H}), 0.88(\mathrm{t}, J$ $=7.3 \mathrm{~Hz}, 3 \mathrm{H})$.

${ }^{13} \mathbf{C}-\mathbf{N M R}\left(\mathbf{C D C l}_{\mathbf{3}}, \mathbf{7 5} \mathbf{M H z}\right): 170.9,145.3,144.8,133.2,129.2,129.0,127.8,127.0,126.4,38.6,35.5$, 33.3, 22.3, 14.0.

HRMS (ESI): $[\mathrm{M}+\mathrm{Na}]^{+}$Calculated for $\mathrm{C}_{18} \mathrm{H}_{21} \mathrm{NONa}$ : 290.1515; Found: 290.1511 . 


\section{Bibliography}

(1) Watson, S. C.; Eastham, J. F. J. Organomet. Chem. 1967, 9, 165.

(2) Brown, H. C.; Subrahmanyam, C.; Hamaoka, T.; Ravindran, N.; Bowman, D. H.; Misumi, S.; Unni, M. K.; Somayaji, V.; Bhat, N. G. The Journal of Organic Chemistry 1989, 54, 6068.

(3) Rossi, R.; Carpita, A.; Quirici, M. G.; Gaudenzi, M. L. Tetrahedron 1982, 38, 631.

(4) (a) Commercon, A.; Normant, J.; Villieras, J. J. Organomet. Chem. 1975, 93, 415. (b) Normant, J. F.; Cahiez, G.; Chuit, C.; Villieras, J. J. Organomet. Chem. 1974, 77, 269.

(5) Normant, J. F.; Chuit, C.; Cahiez, G.; Villiera.J Synthesis 1974, 803.

(6) Echavarren, A. M.; Stille, J. K. J. Am. Chem. Soc. 1987, 109, 5478.

(7) Huang, Z.; Liu, Z.; Zhou, J. J. Am. Chem. Soc. 2011, 133, 15882.

(8) Schade, M. A.; Metzger, A.; Hug, S.; Knochel, P. Chem. Commun. 2008, 3046.

(9) Singh, M.; Kumar, S.; Kumar, A.; Kumar, P.; Narasimhan, B. Med. Chem. Res. 2012, 21, 511.

(10) Belger, C.; Neisius, N. M.; Plietker, B. Chemistry - A European Journal 2010, 16, 12214.

(11) Hodgson, D. M.; Fleming, M. J.; Stanway, S. J. The Journal of Organic Chemistry 2007, 72, 4763.

(12) Brown, H. C.; Basavaiah, D.; Kulkarni, S. U.; Bhat, N. G.; Prasad, J. V. N. V. The Journal of Organic Chemistry 1988, 53, 239.

(13) Cahiez, G.; Avedissian, H. Synthesis 1998, 1199.

(14) Cahiez, G.; Duplais, C.; Moyeux, A. Org. Lett. 2007, 9, 3253.

(15) Cahiez, G.; Gager, O.; Buendia, J.; Patinote, C. Chem. - Eur. J. 2012, 18, 5860.

(16) Ciganek, E. The Journal of Organic Chemistry 1992, 57, 4521.

(17) Kazuhiko, T.; Mitsuyoshi, S.; Koichiro, O.; Hitosi, N. Bull. Chem. Soc. Jpn. 1984, 57, 108.

(18) Fürstner, A.; Leitner, A.; Méndez, M.; Krause, H. J. Am. Chem. Soc. 2002, 124, 13856.

(19) Piontek, A.; Szostak, M. Eur. J. Org. Chem. 2017, 2017, 7271.

(20) Kondolff, I.; Doucet, H.; Santelli, M. Tetrahedron 2004, 60, 3813.

(21) Tsuchiya, Y.; Morita, O.; Tsukamoto, Y.; Okada, H. Yakugaku zasshi journal of the Pharmaceutical Society of Japan 1976, 96, 1005.

(22) Martinelli, J. R.; Clark, T. P.; Watson, D. A.; Munday, R. H.; Buchwald, S. L. Angew. Chem. Int. Ed. 2007, 46, 8460 . 
${ }^{1} \mathrm{H}$ and ${ }^{13} \mathrm{C}$ NMR spectra of synthesized compounds
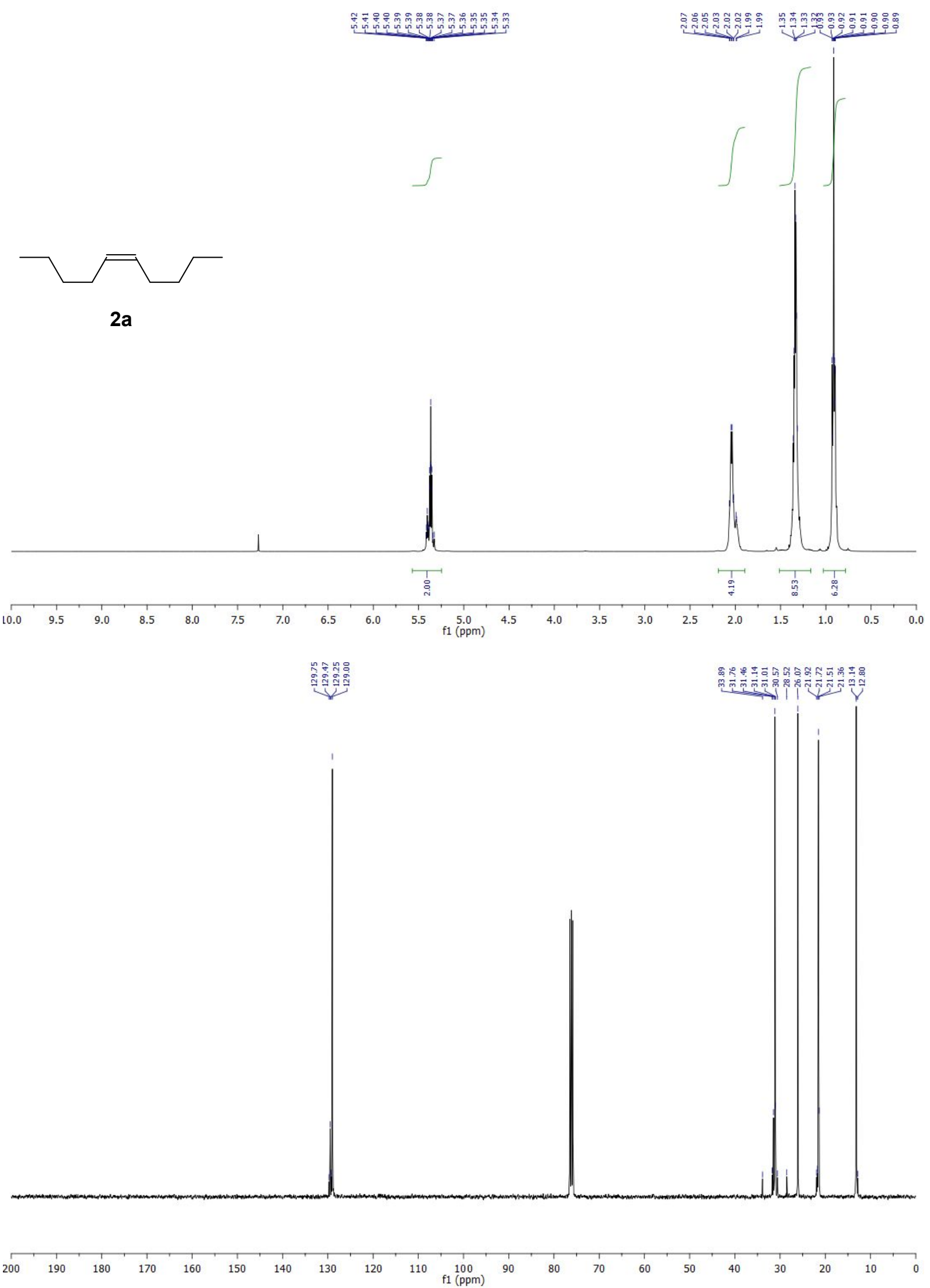


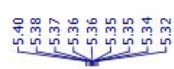

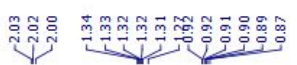

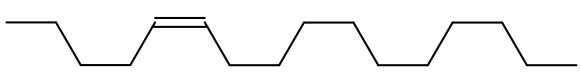

2b

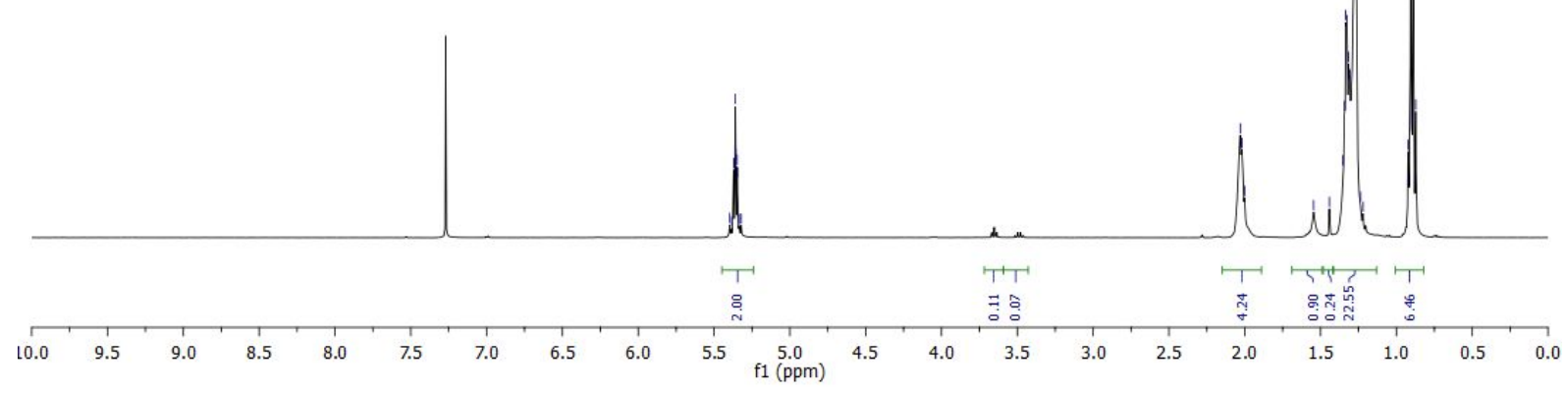

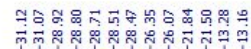

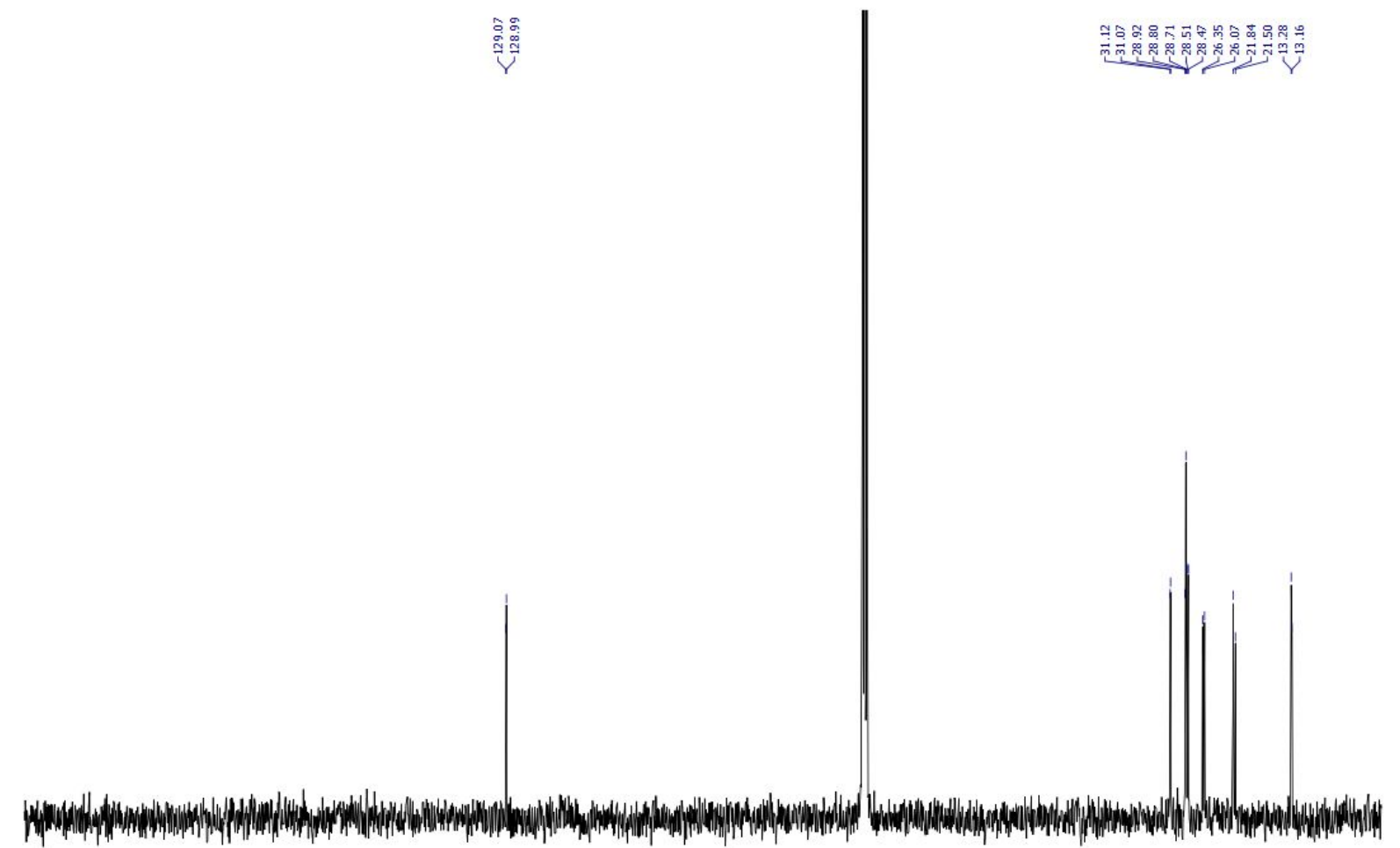

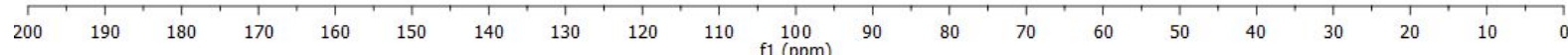




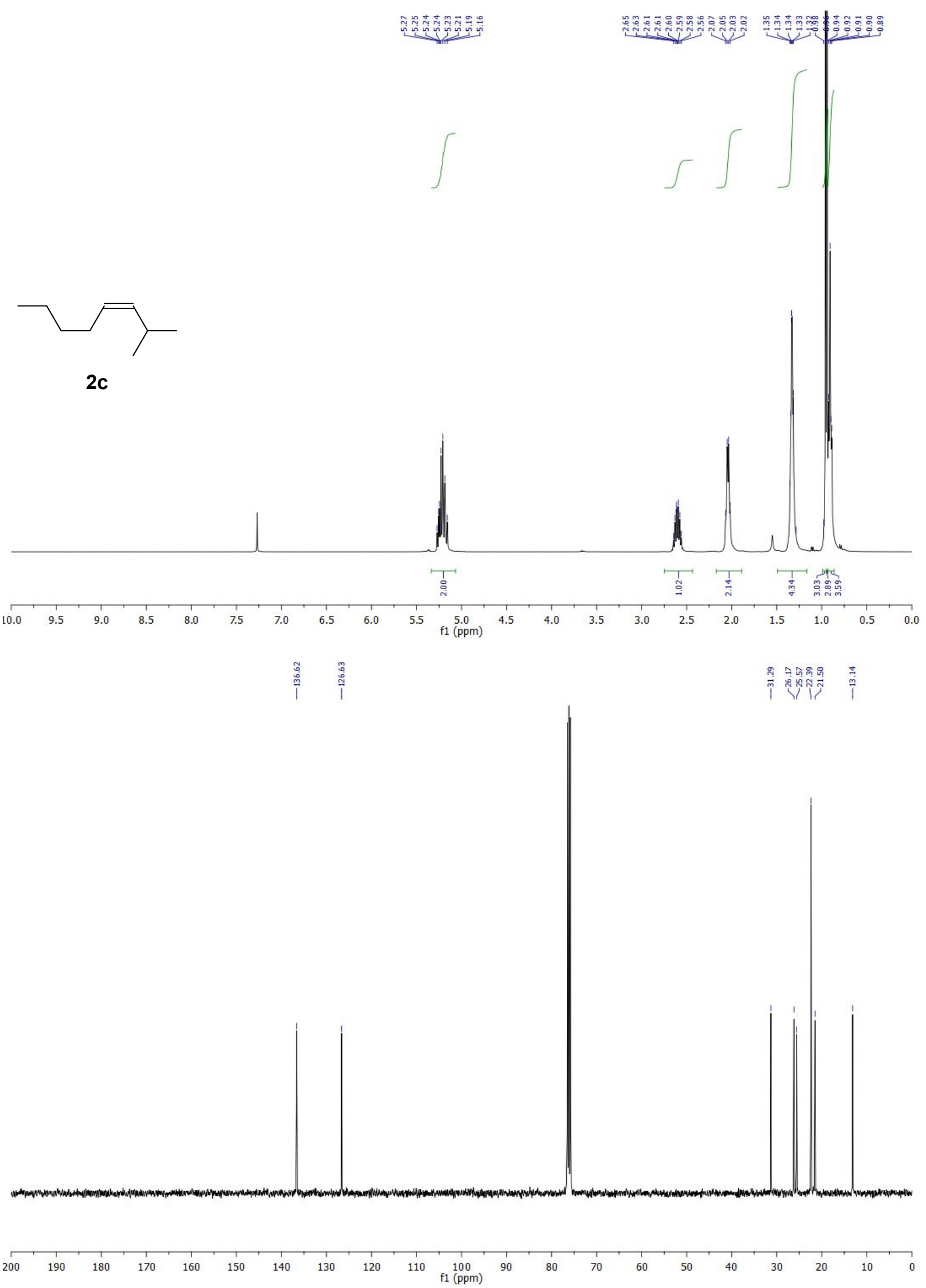



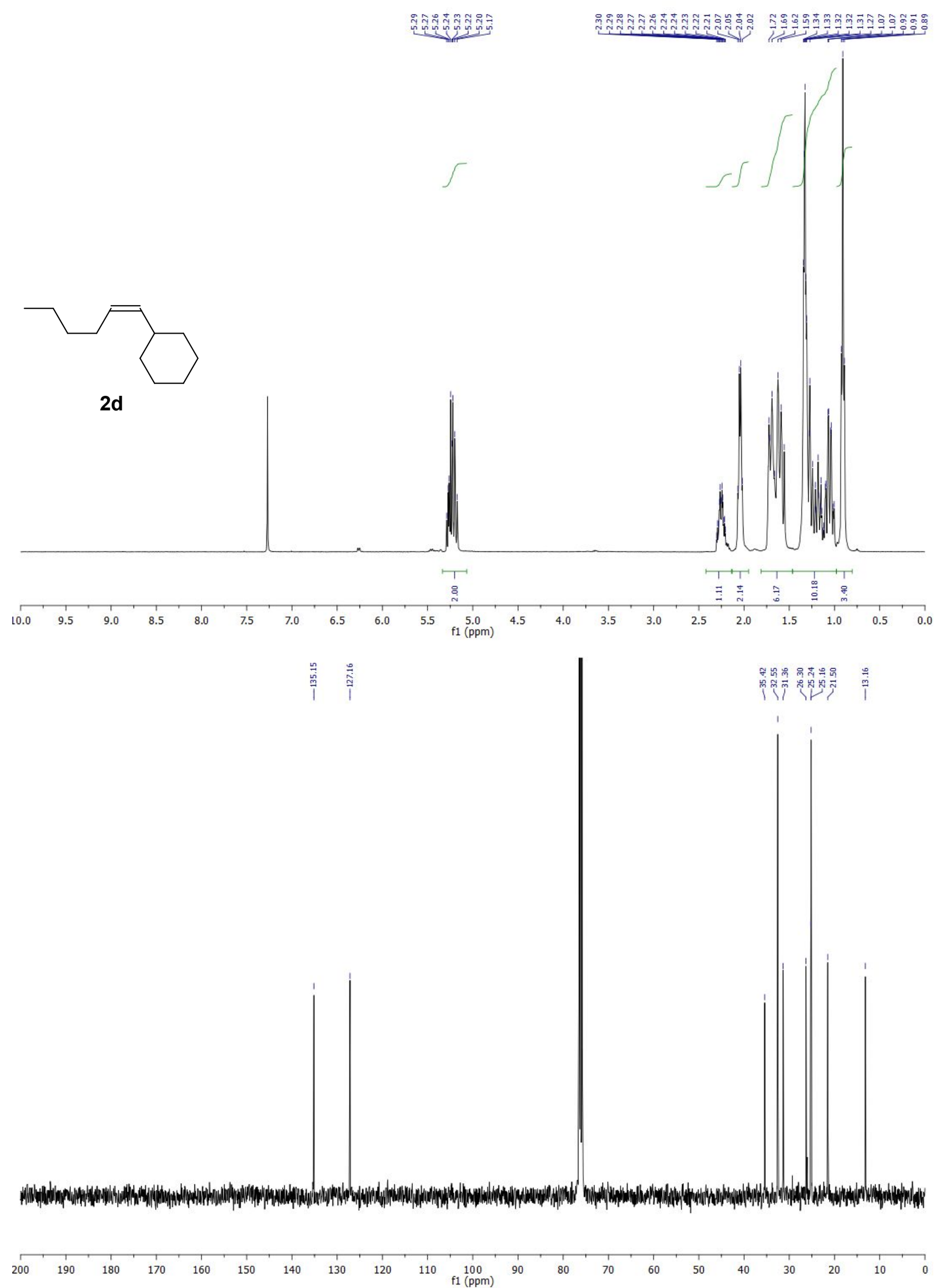

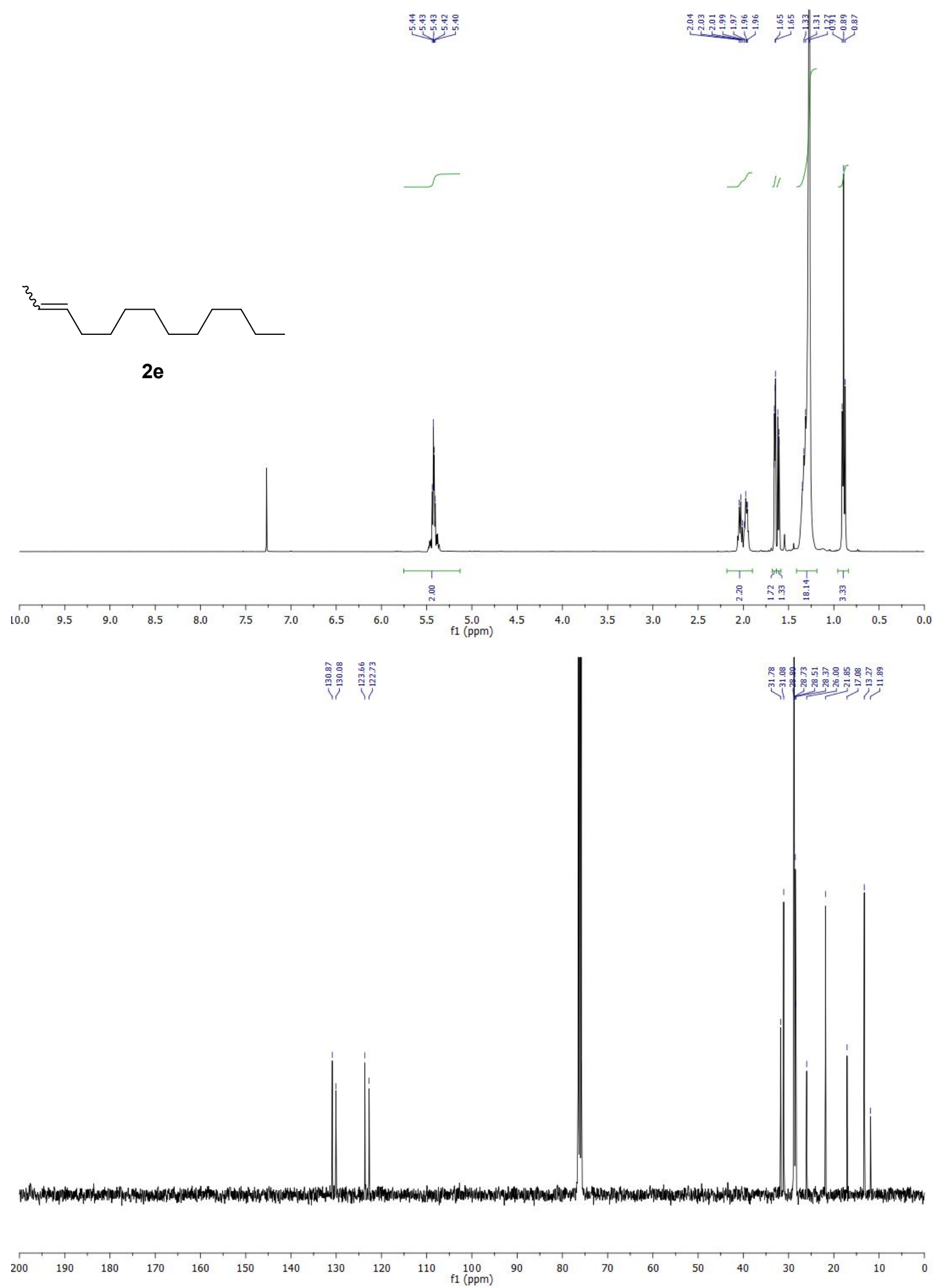


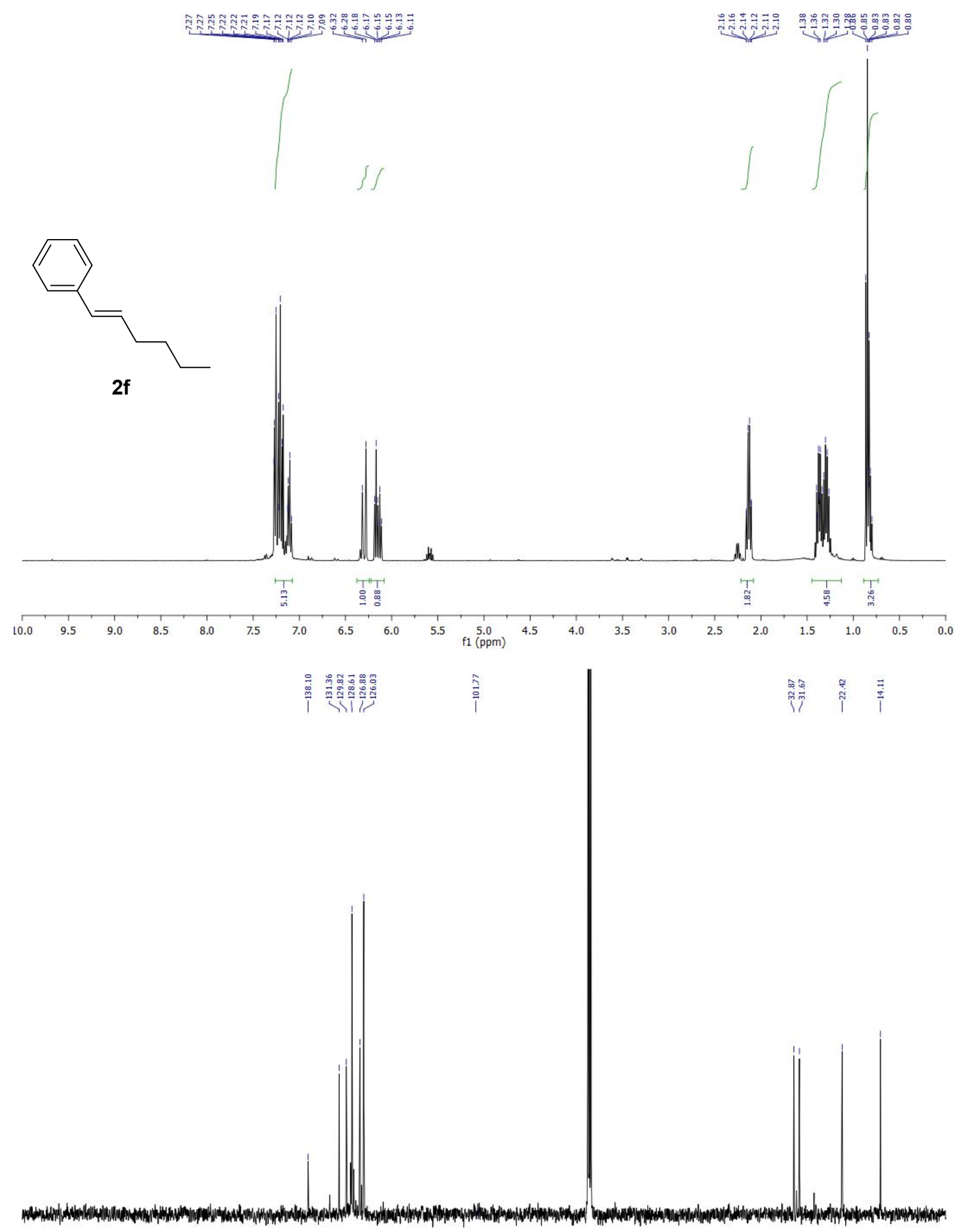

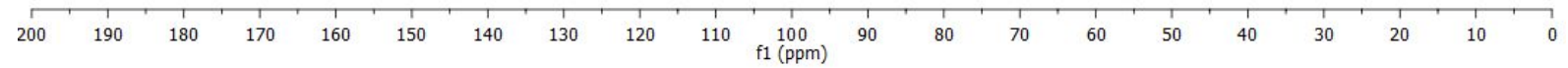



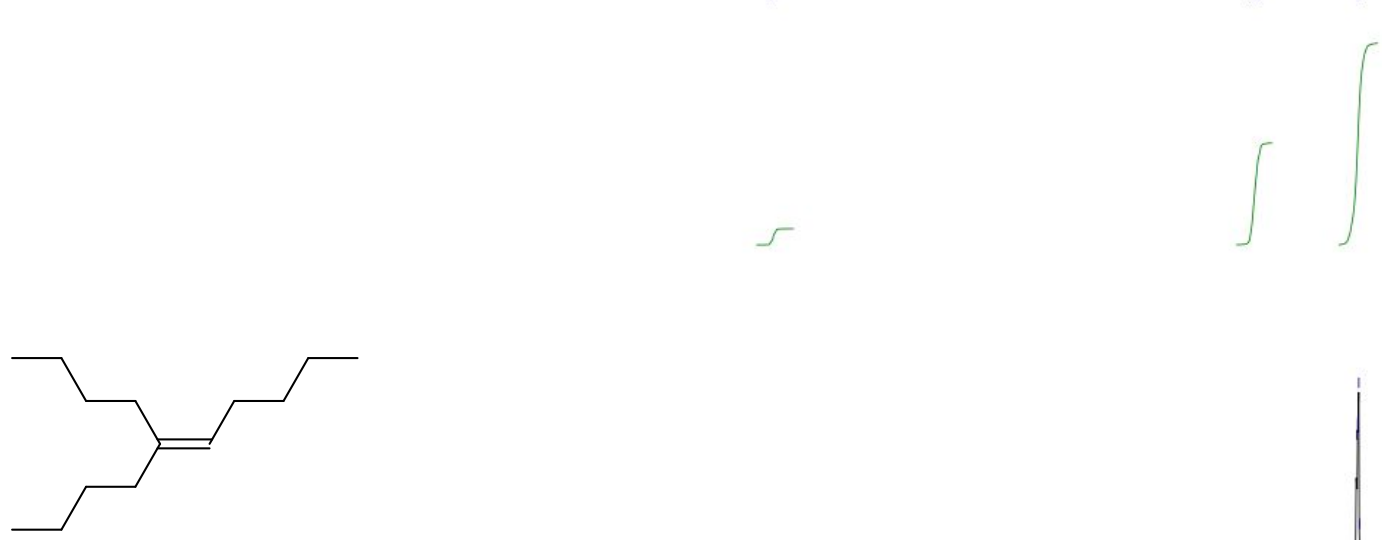

$2 g$

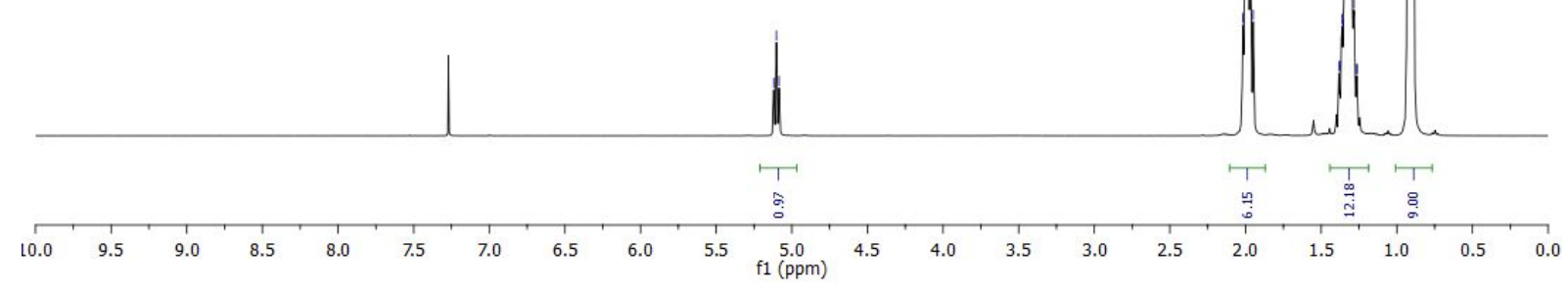

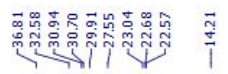

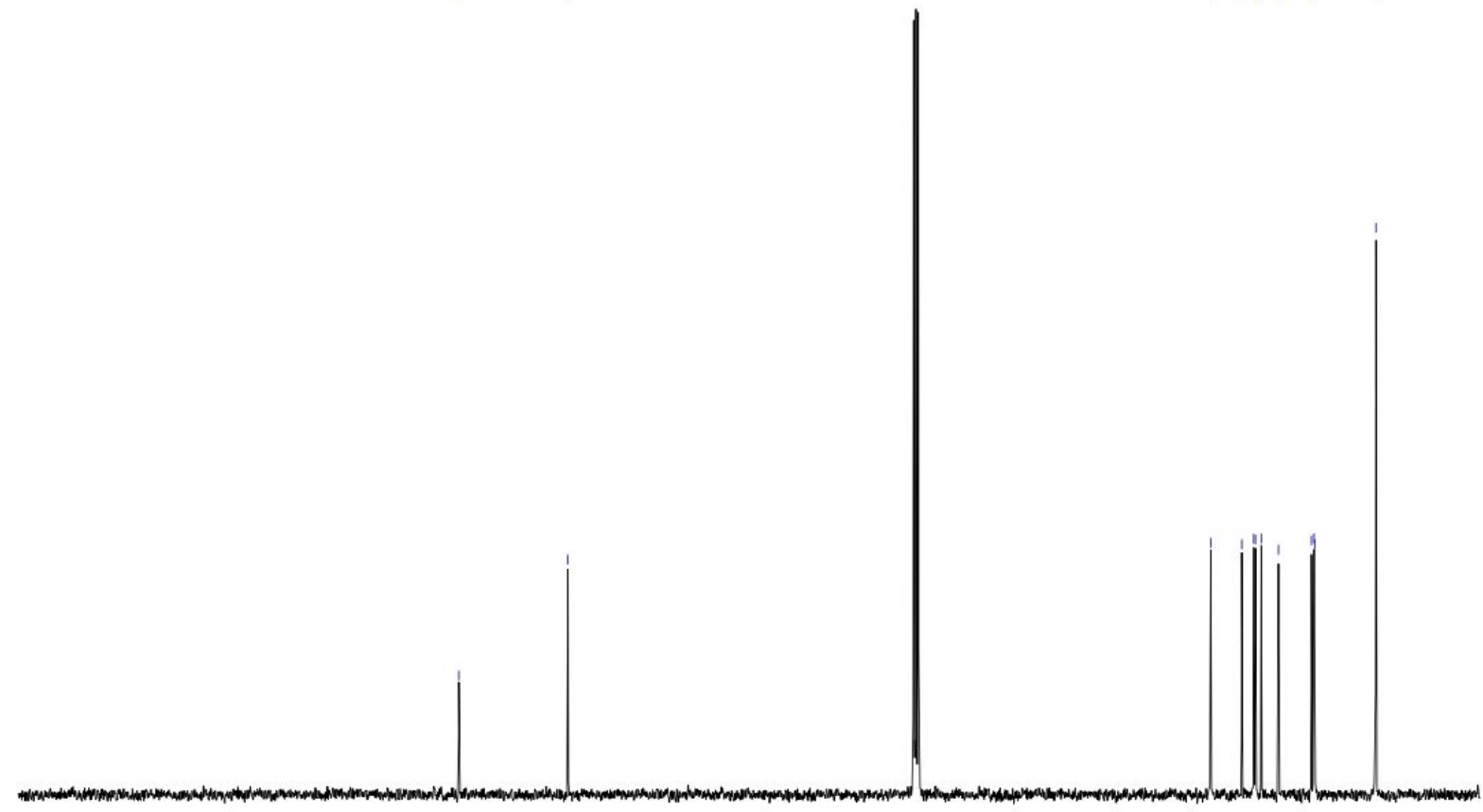

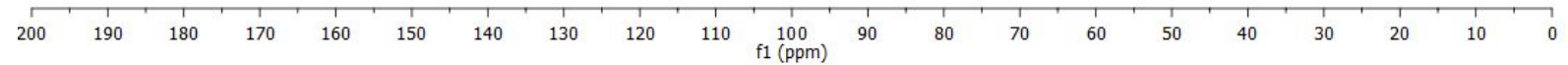




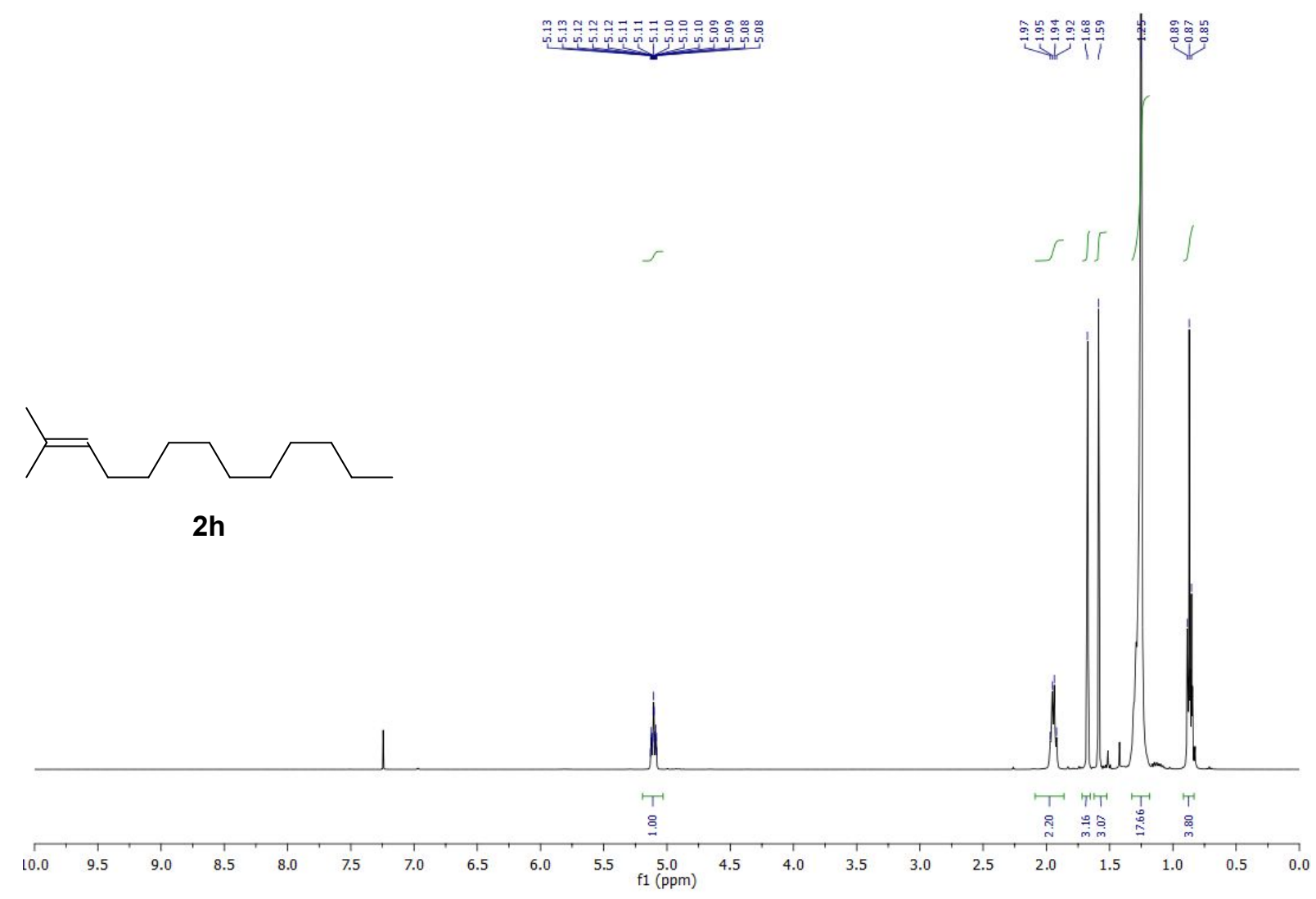

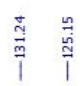

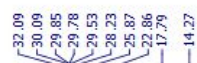

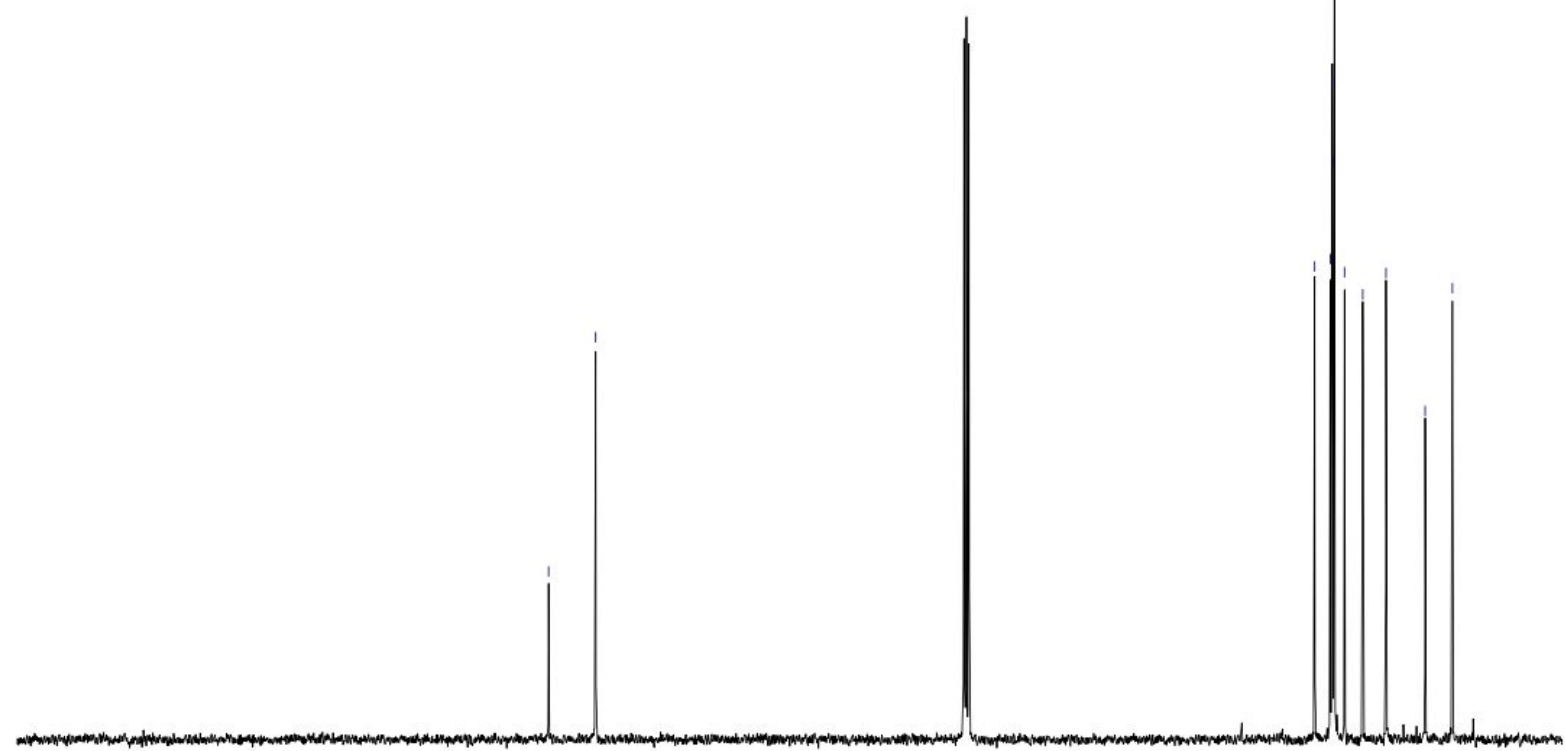

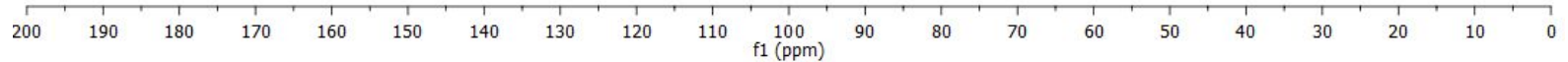



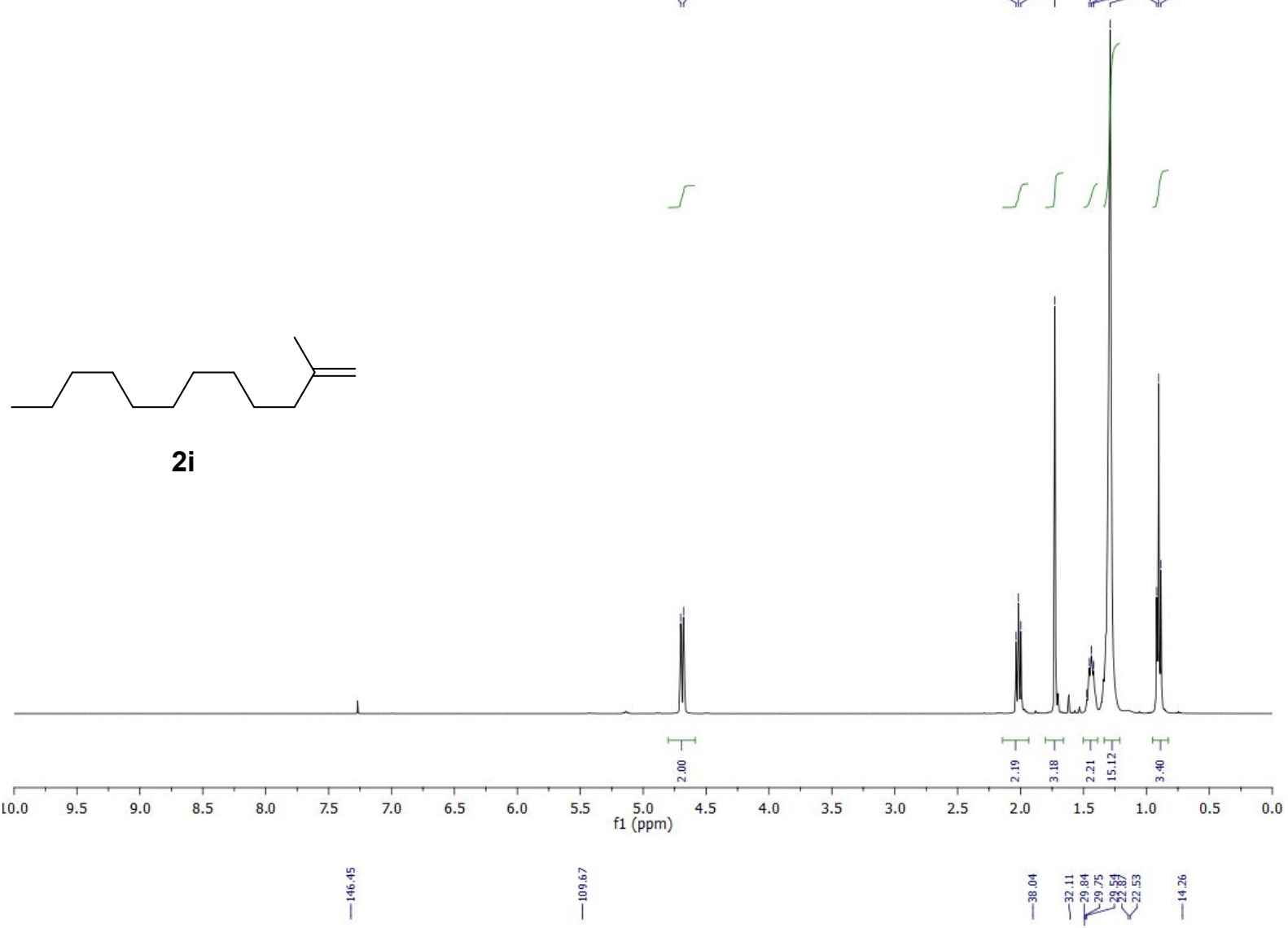

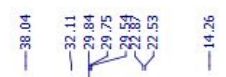

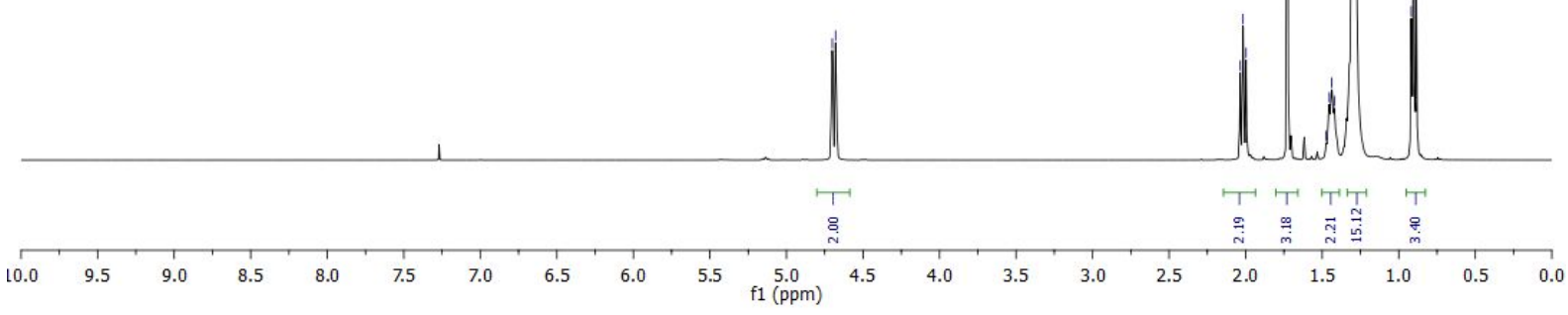
i

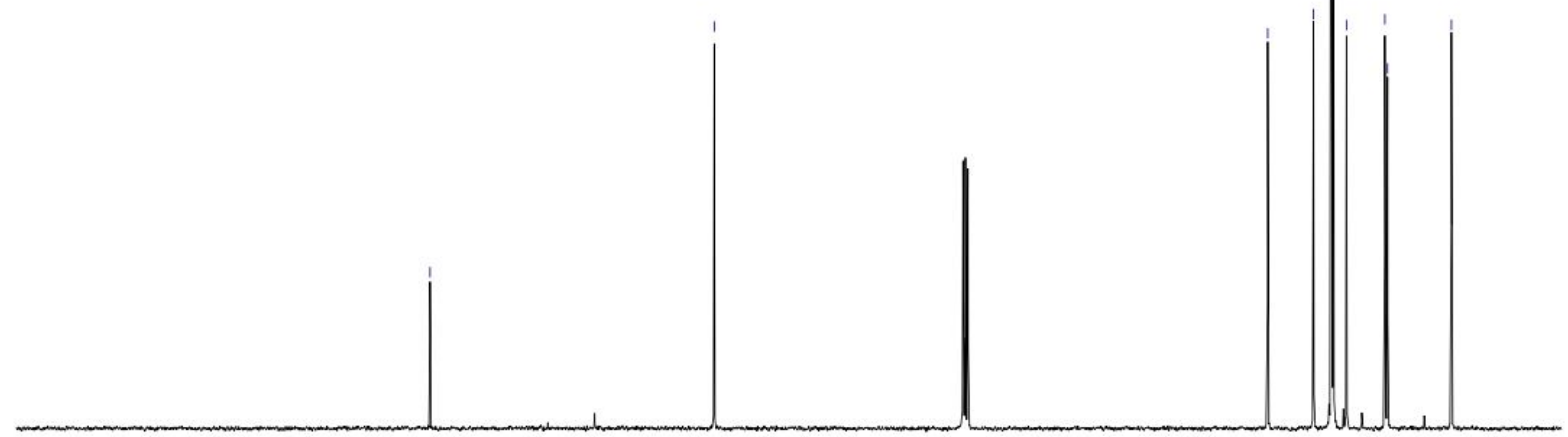

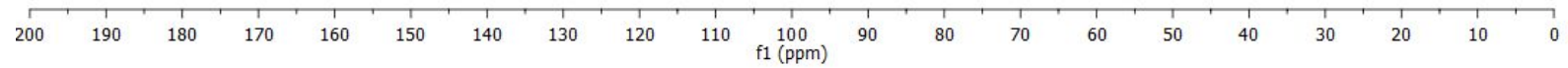



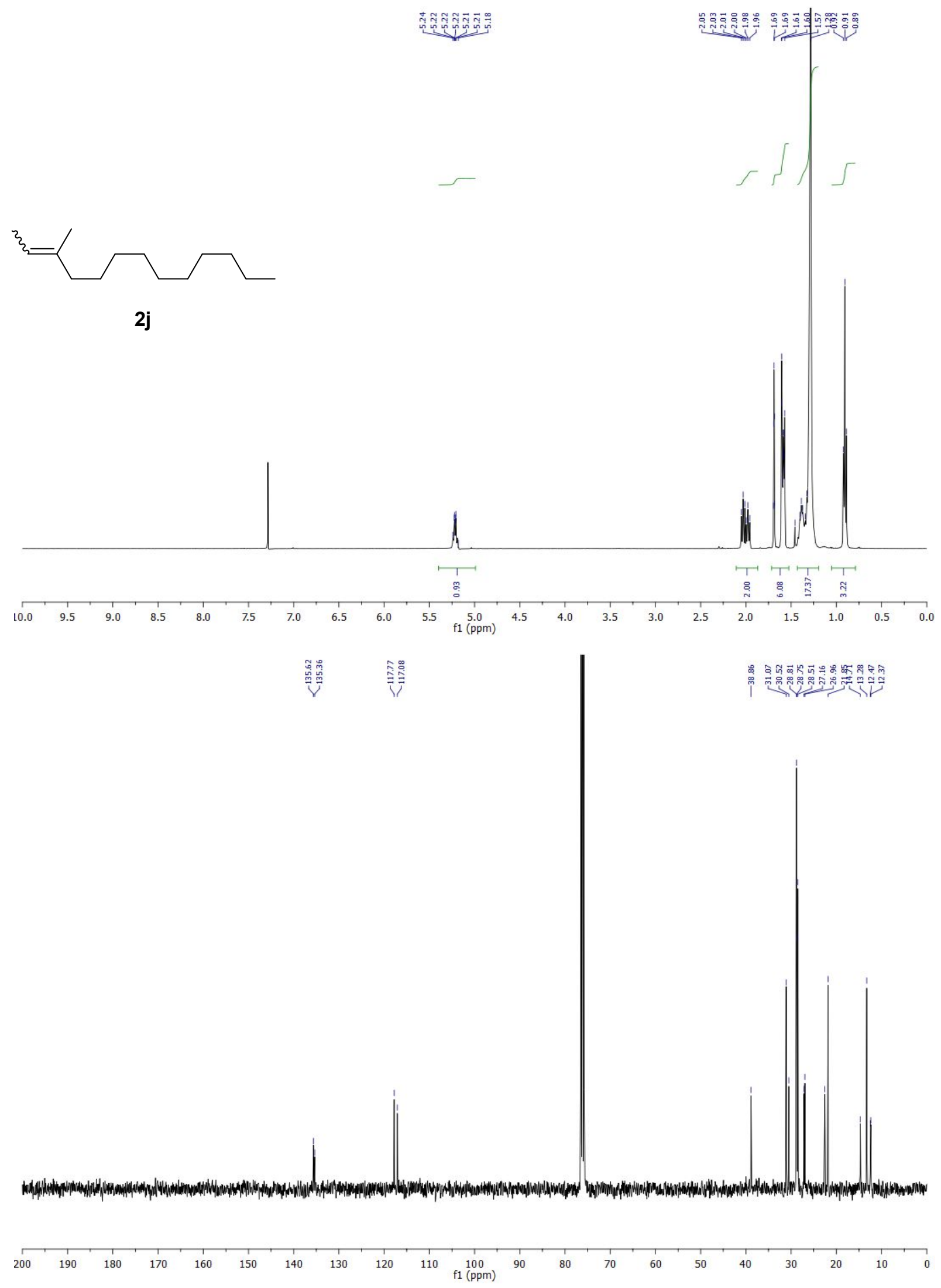


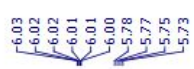

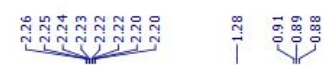

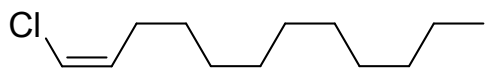

$2 k$

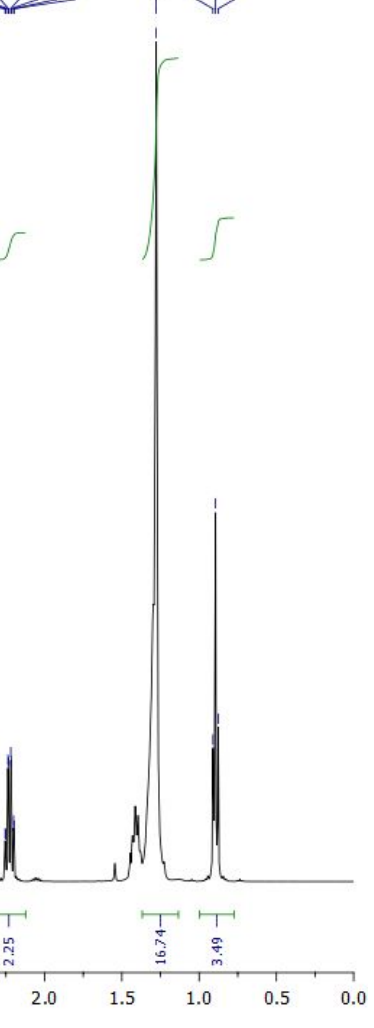

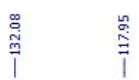

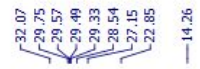

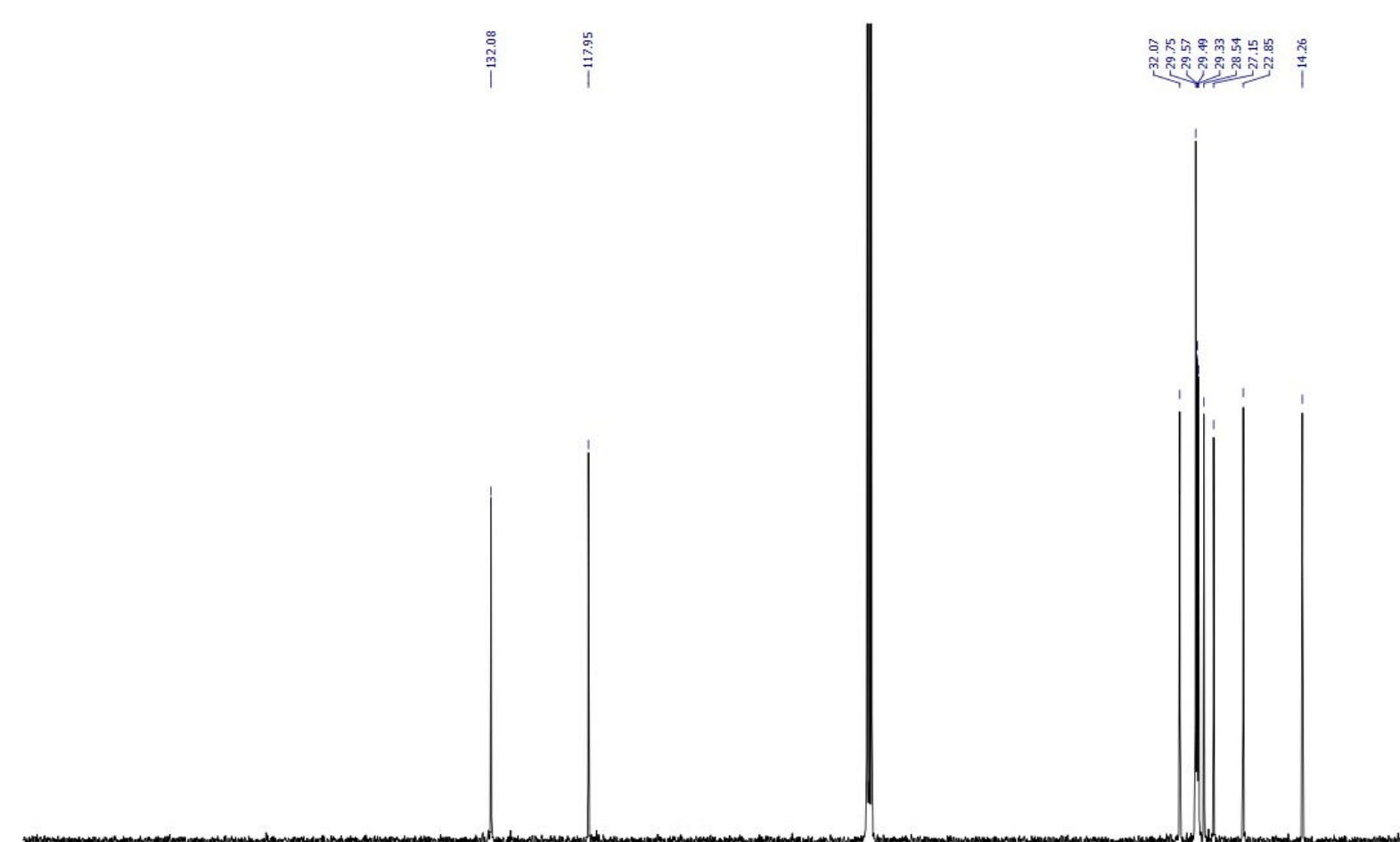

$\begin{array}{lllllllllllllllllllllllllllllll}200 & 190 & 180 & 170 & 160 & 150 & 140 & 130 & 120 & 110 & 100 & 90 & 80 & 70 & 60 & 50 & 40 & 30 & 20 & 10 & 0\end{array}$ 


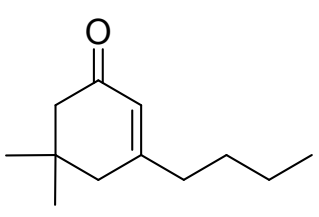

2I
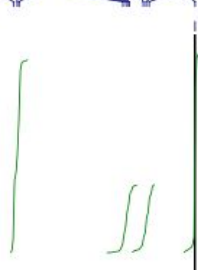

in.
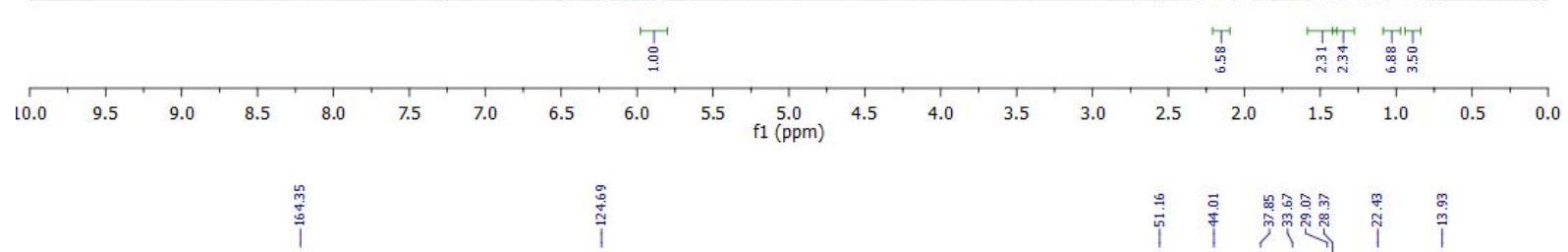

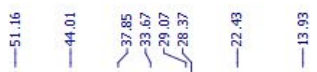

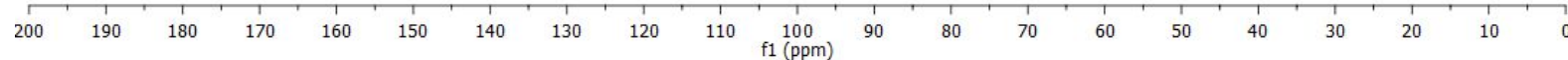



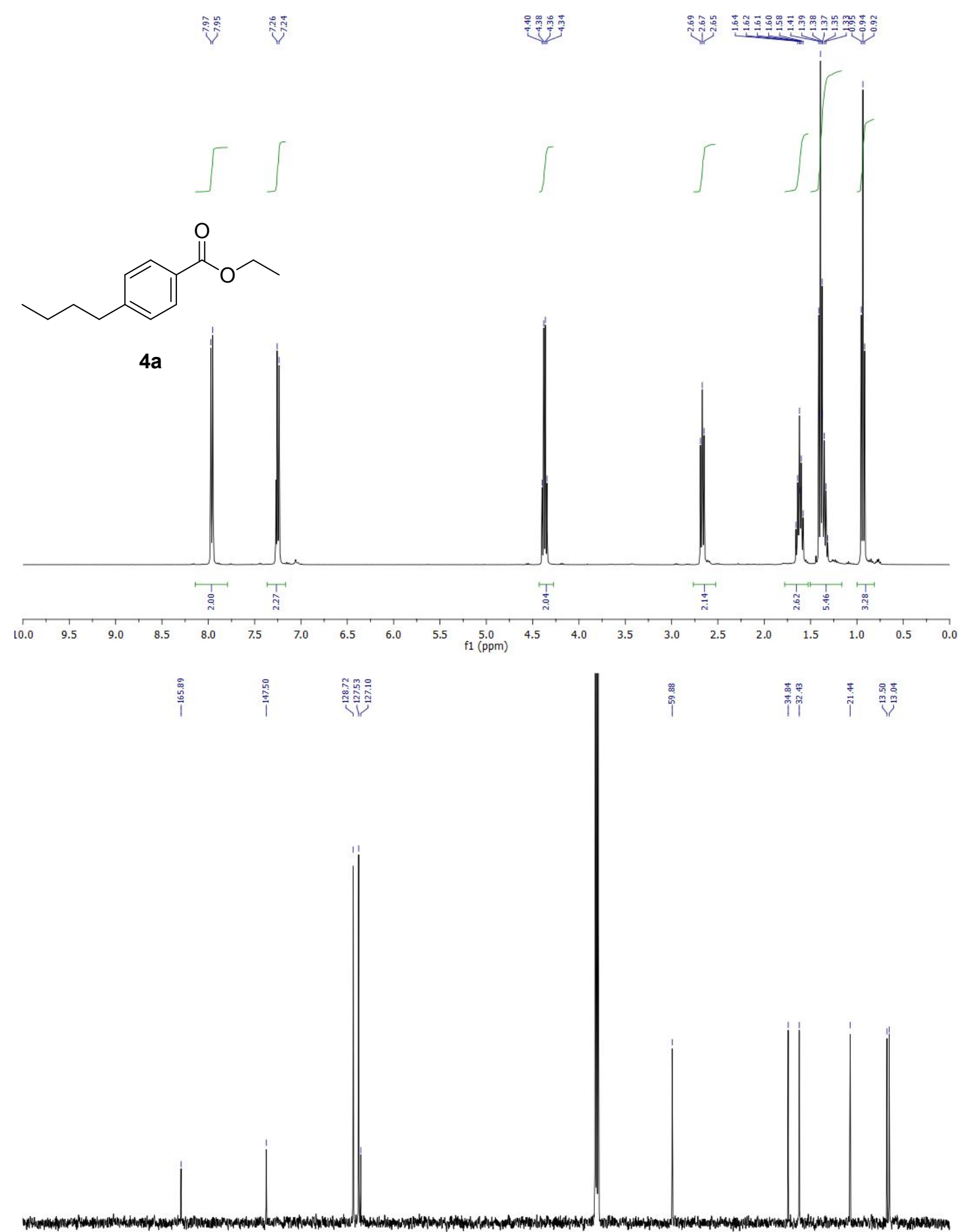

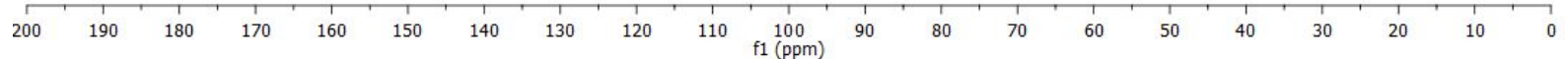



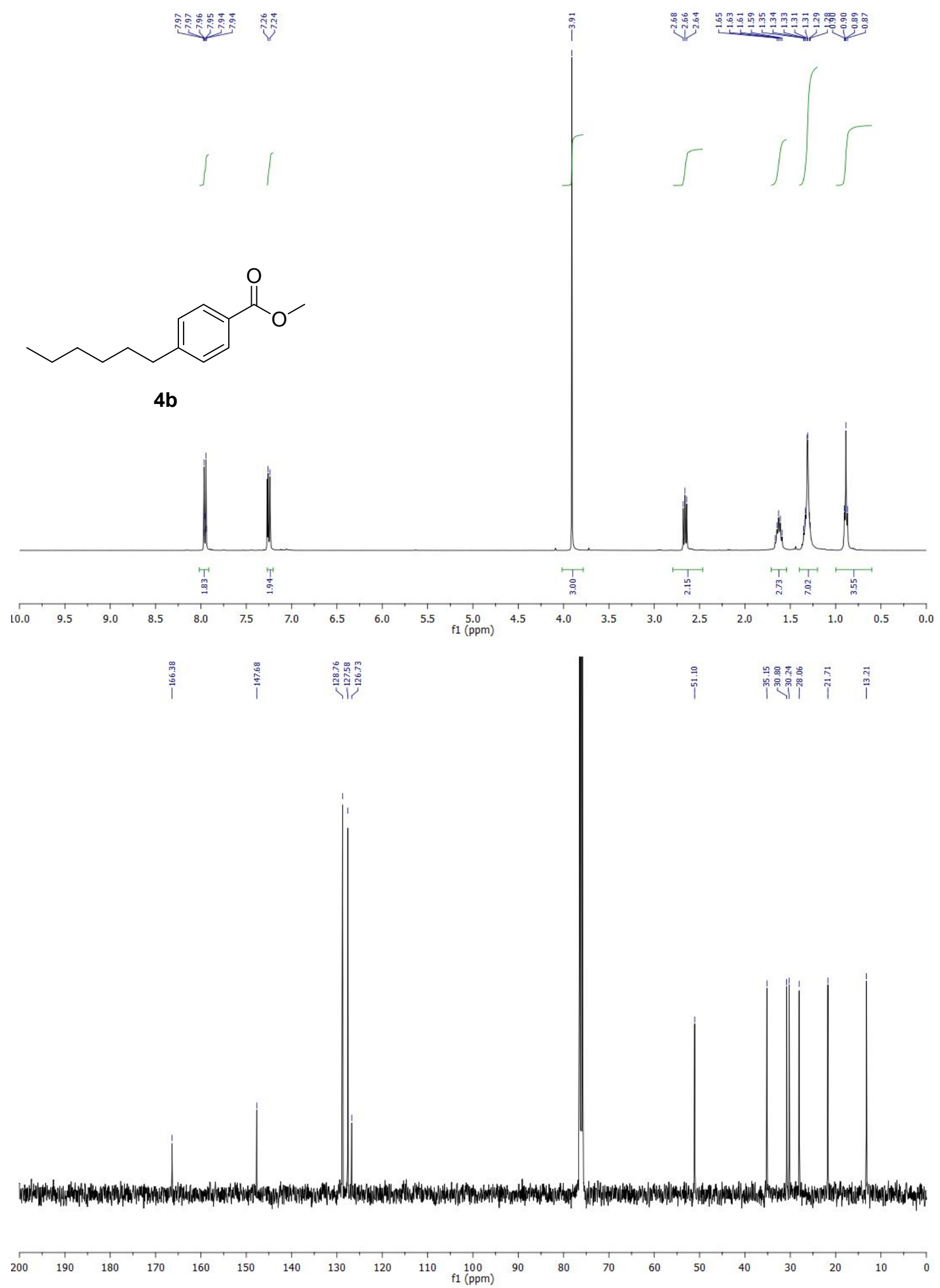

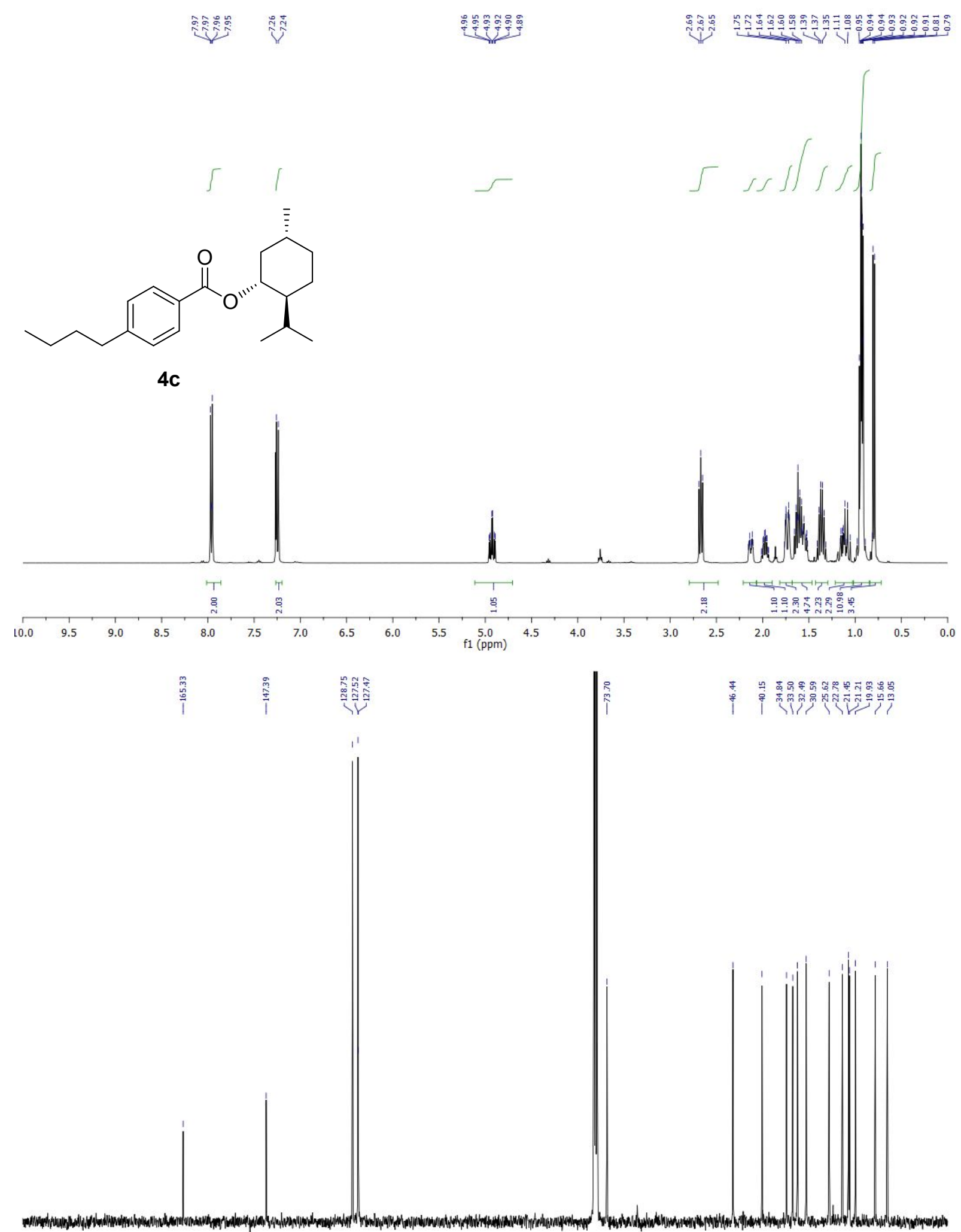

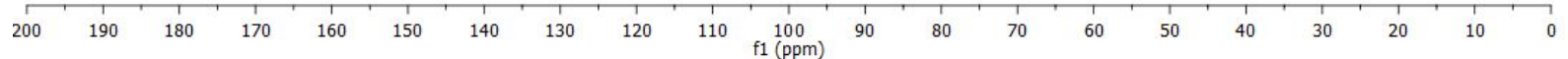


<smiles>CCCCc1ccc2ccccc2c1</smiles>

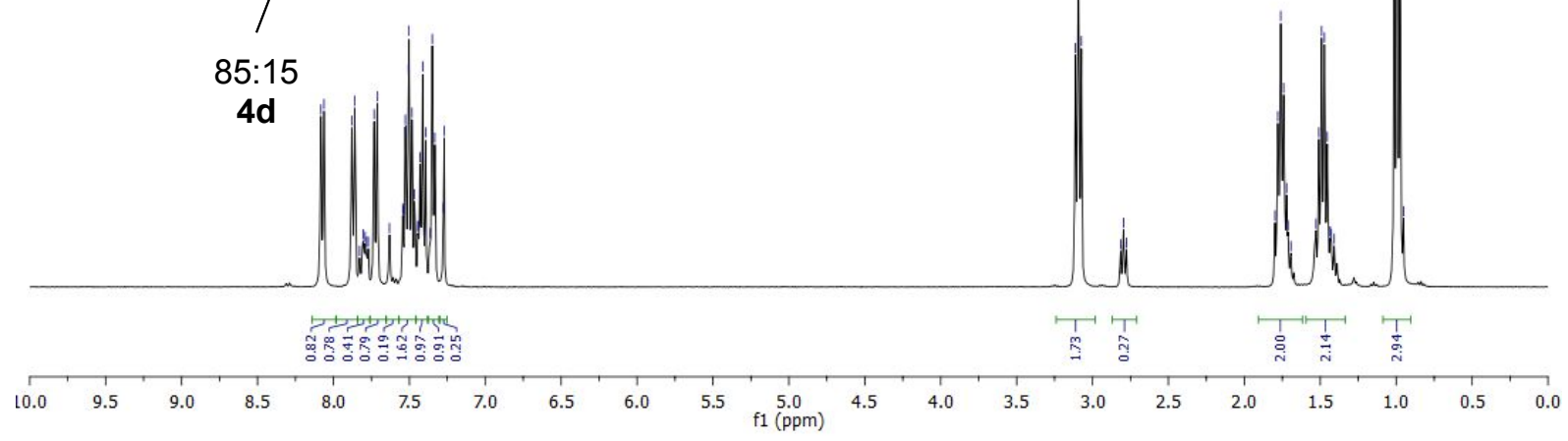

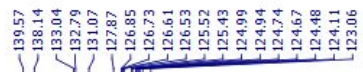

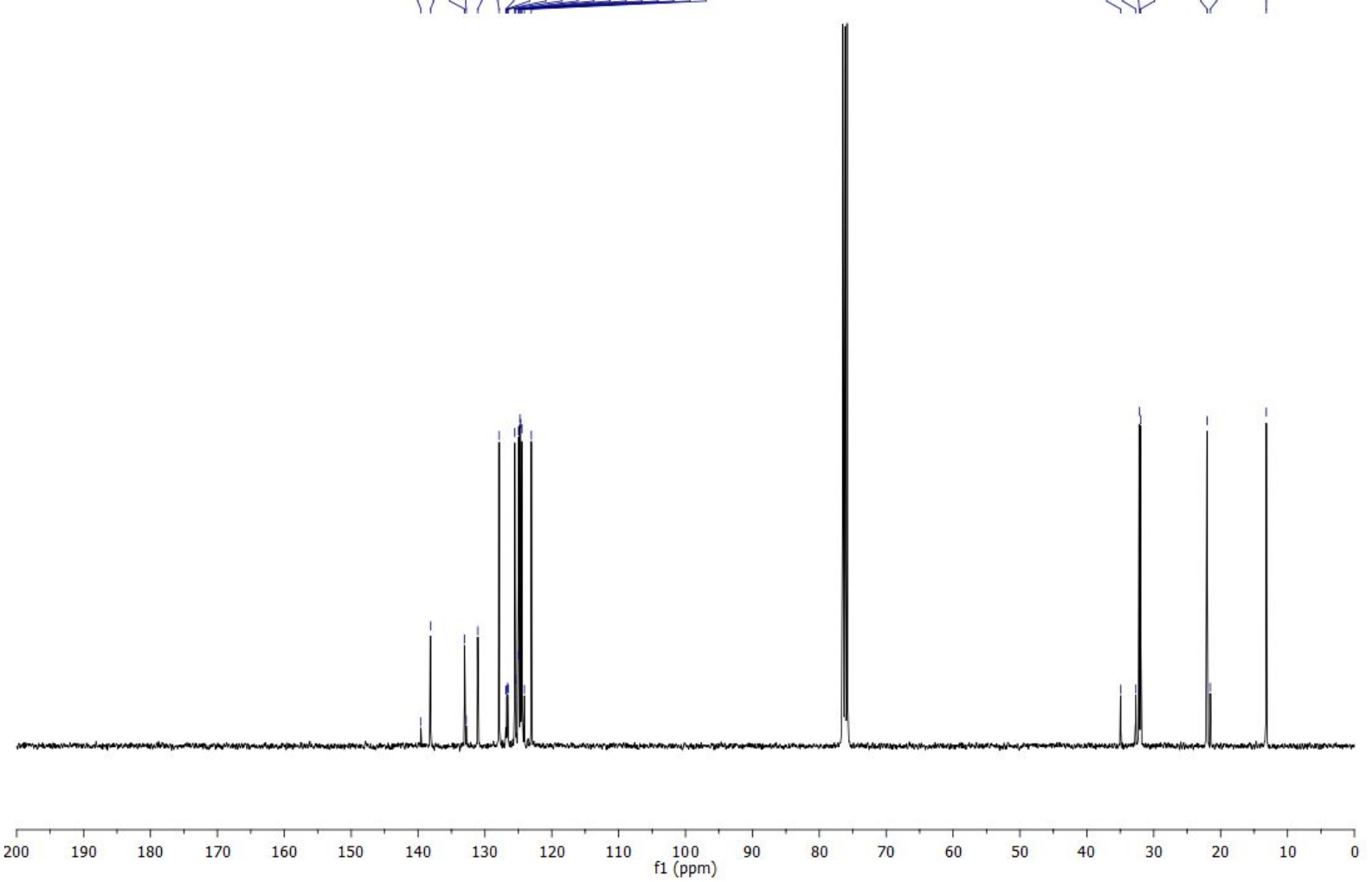



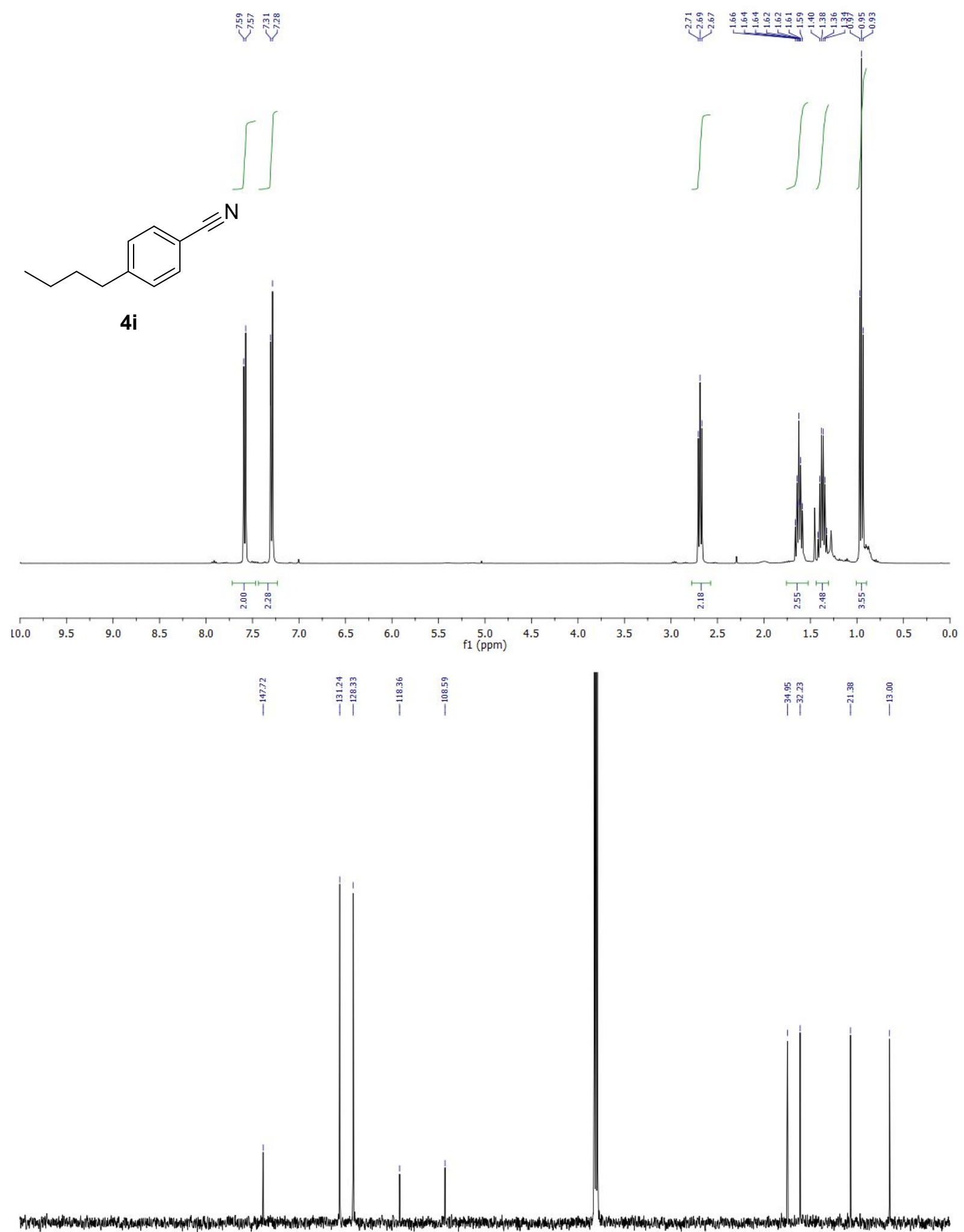

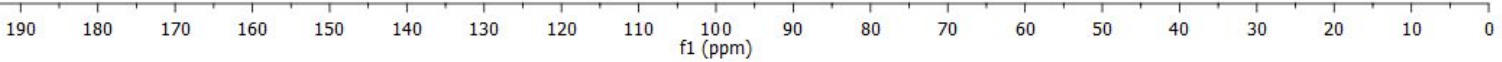




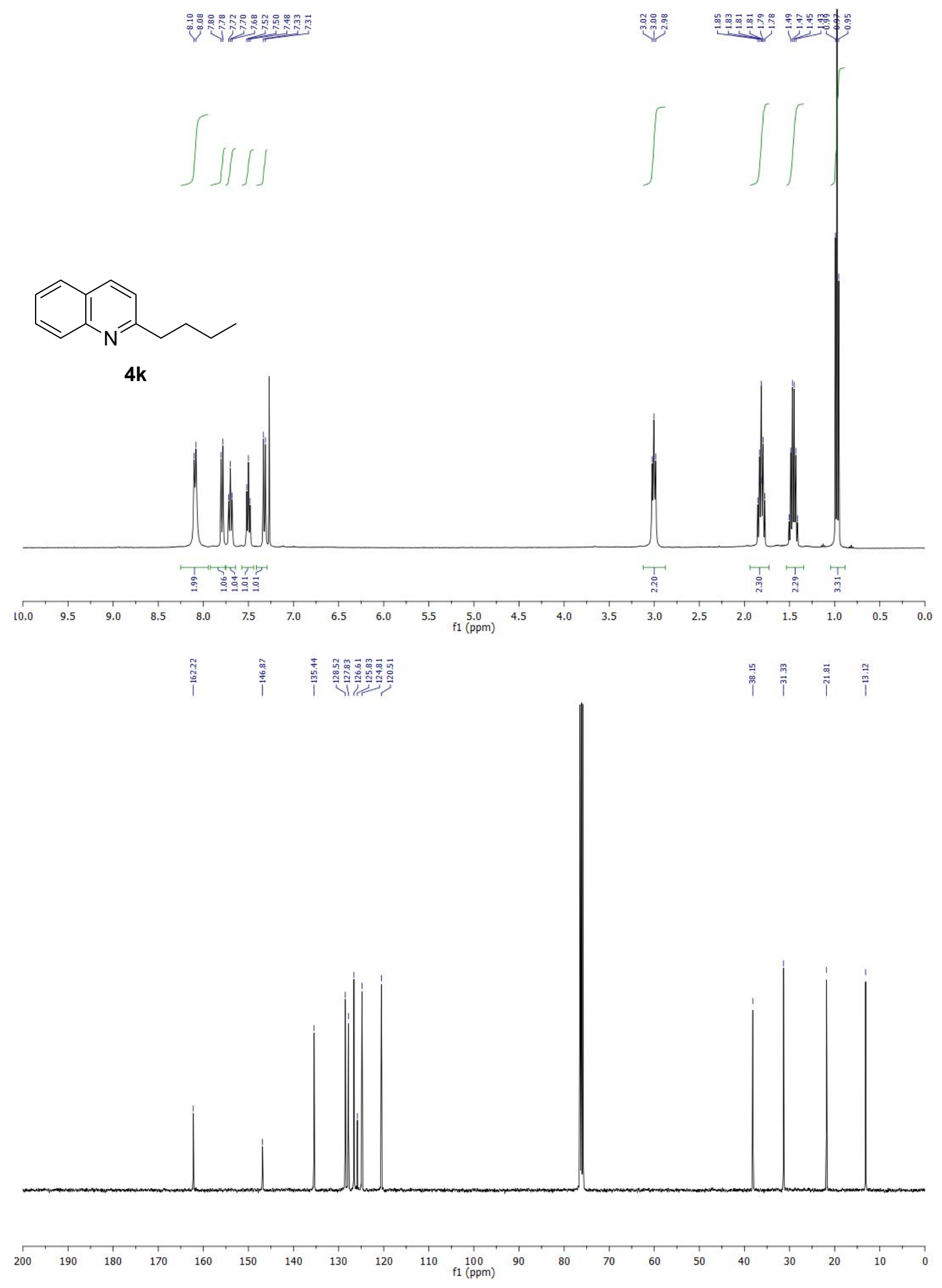



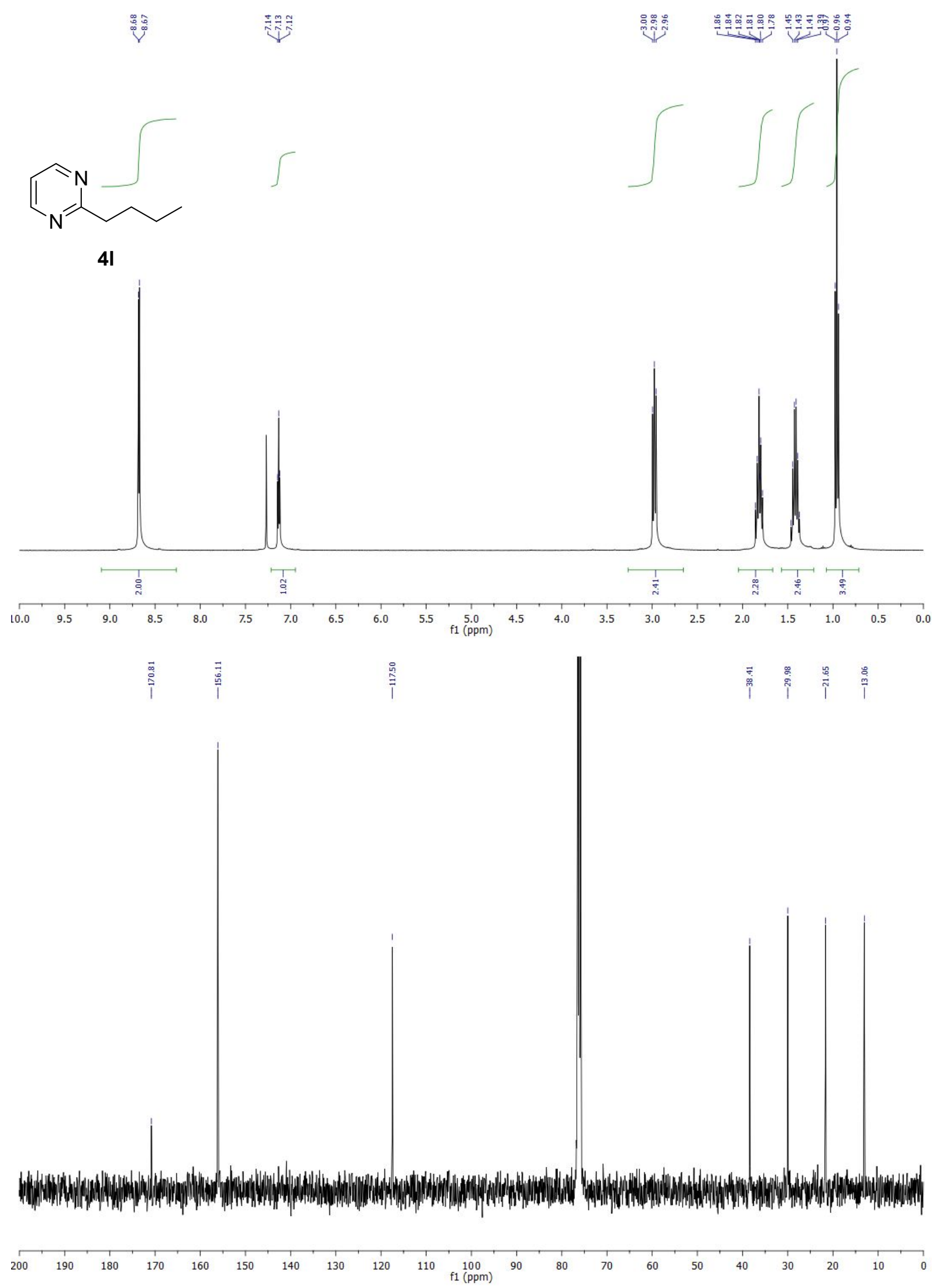

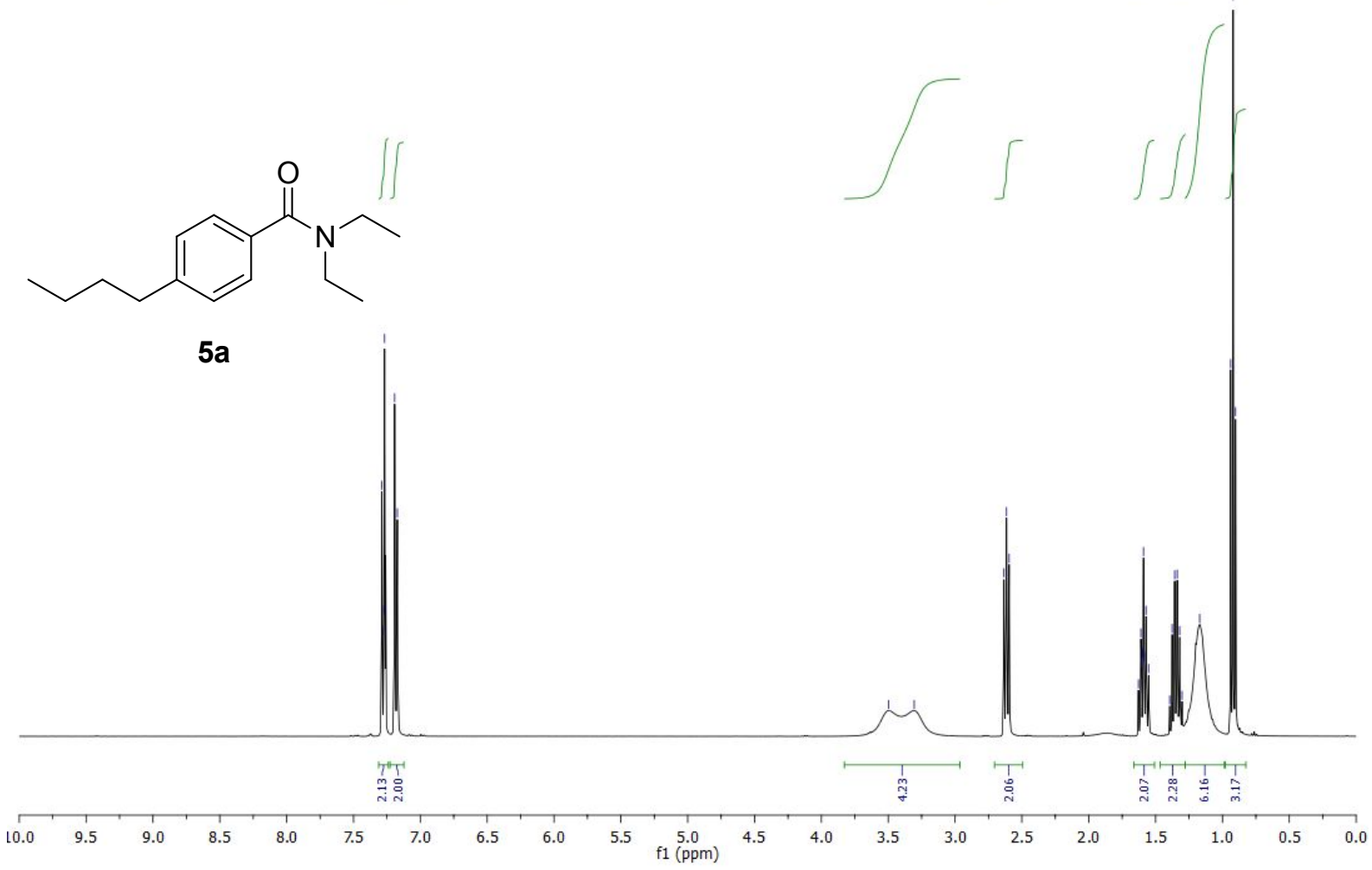

ใิ้

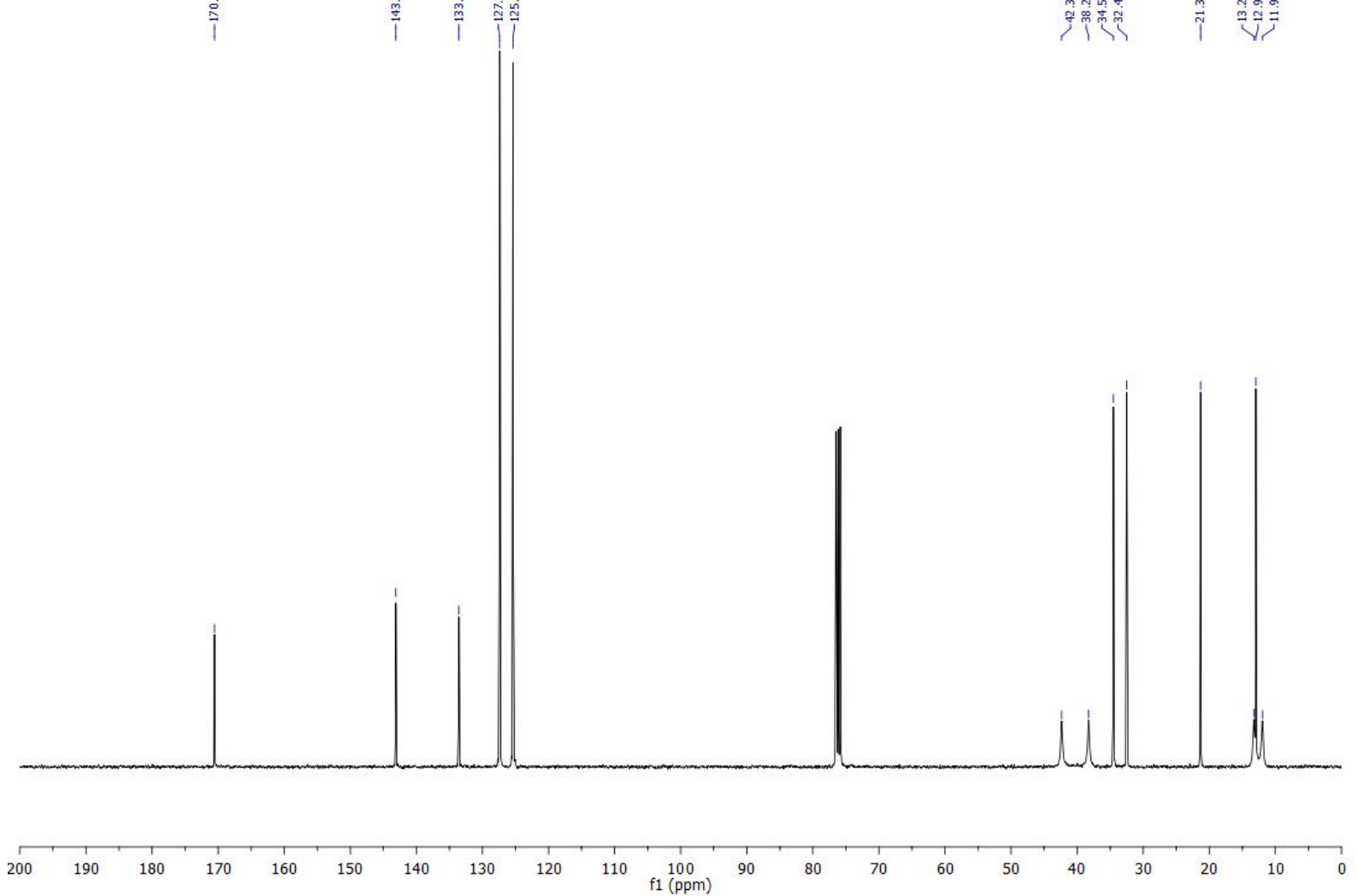




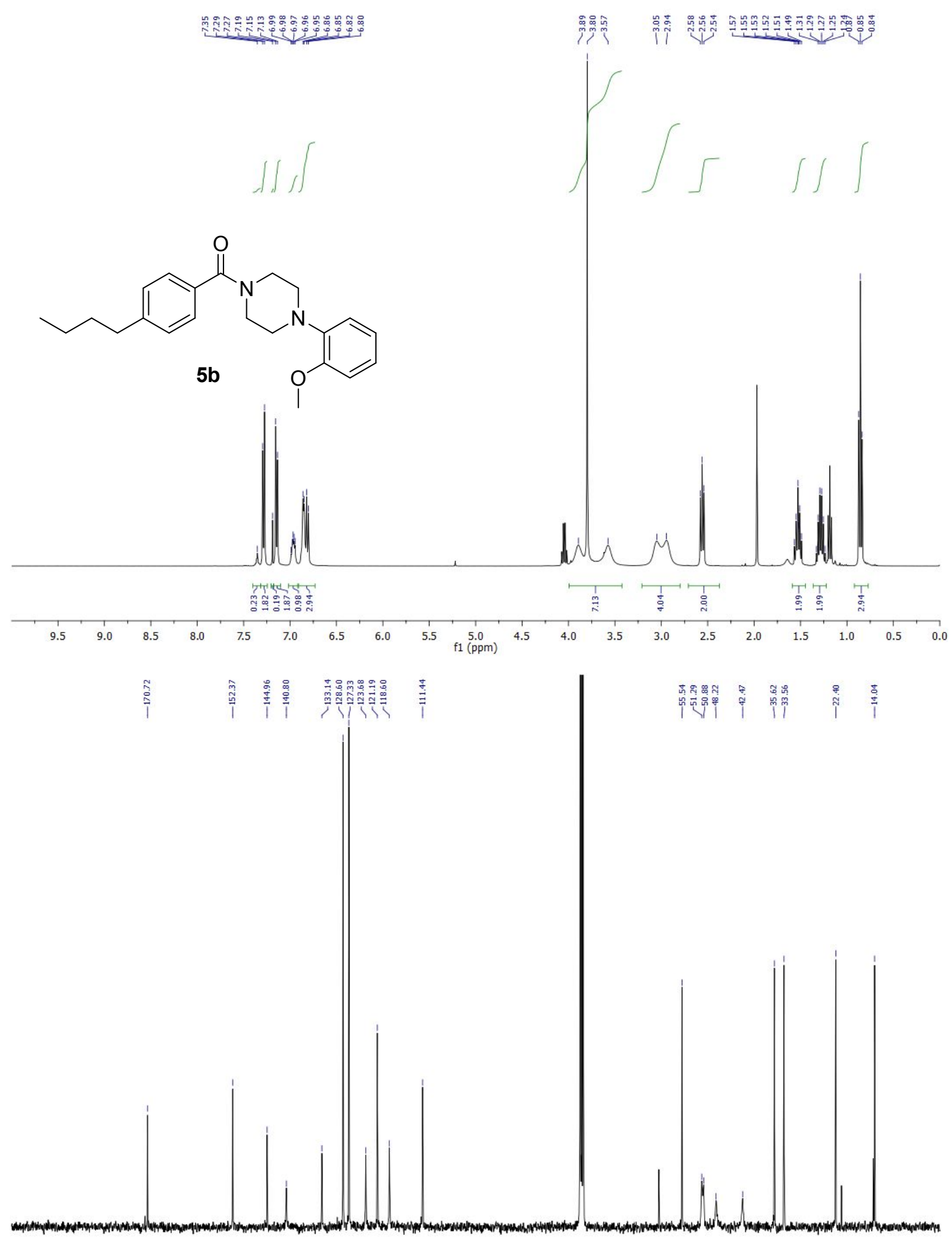

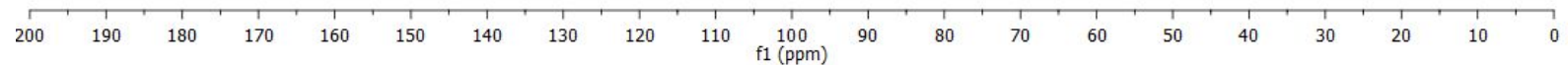




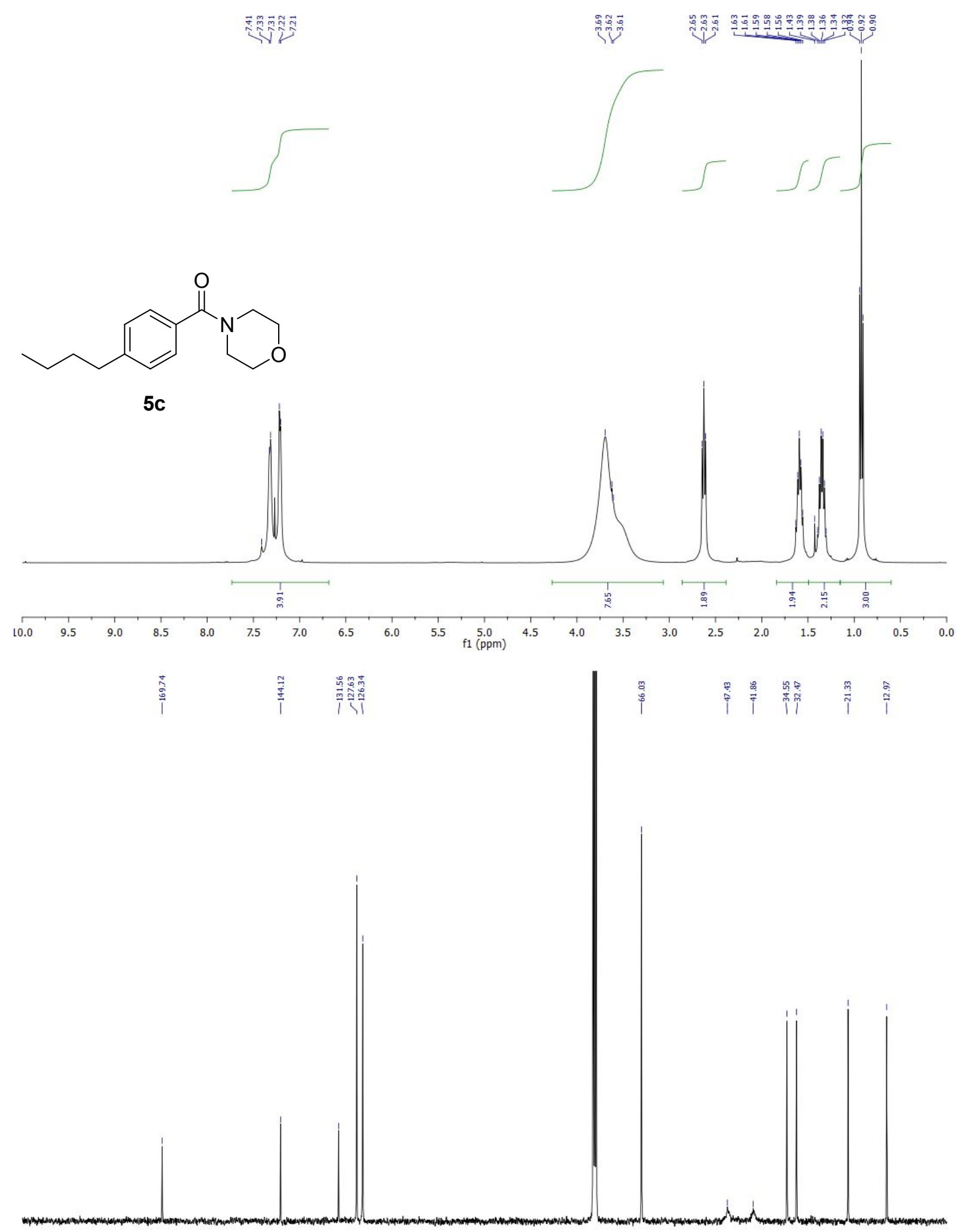

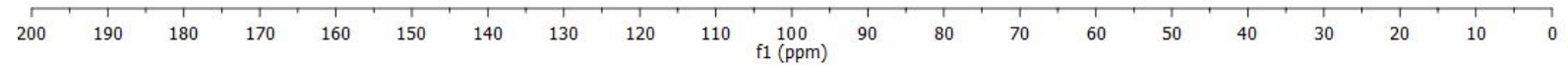




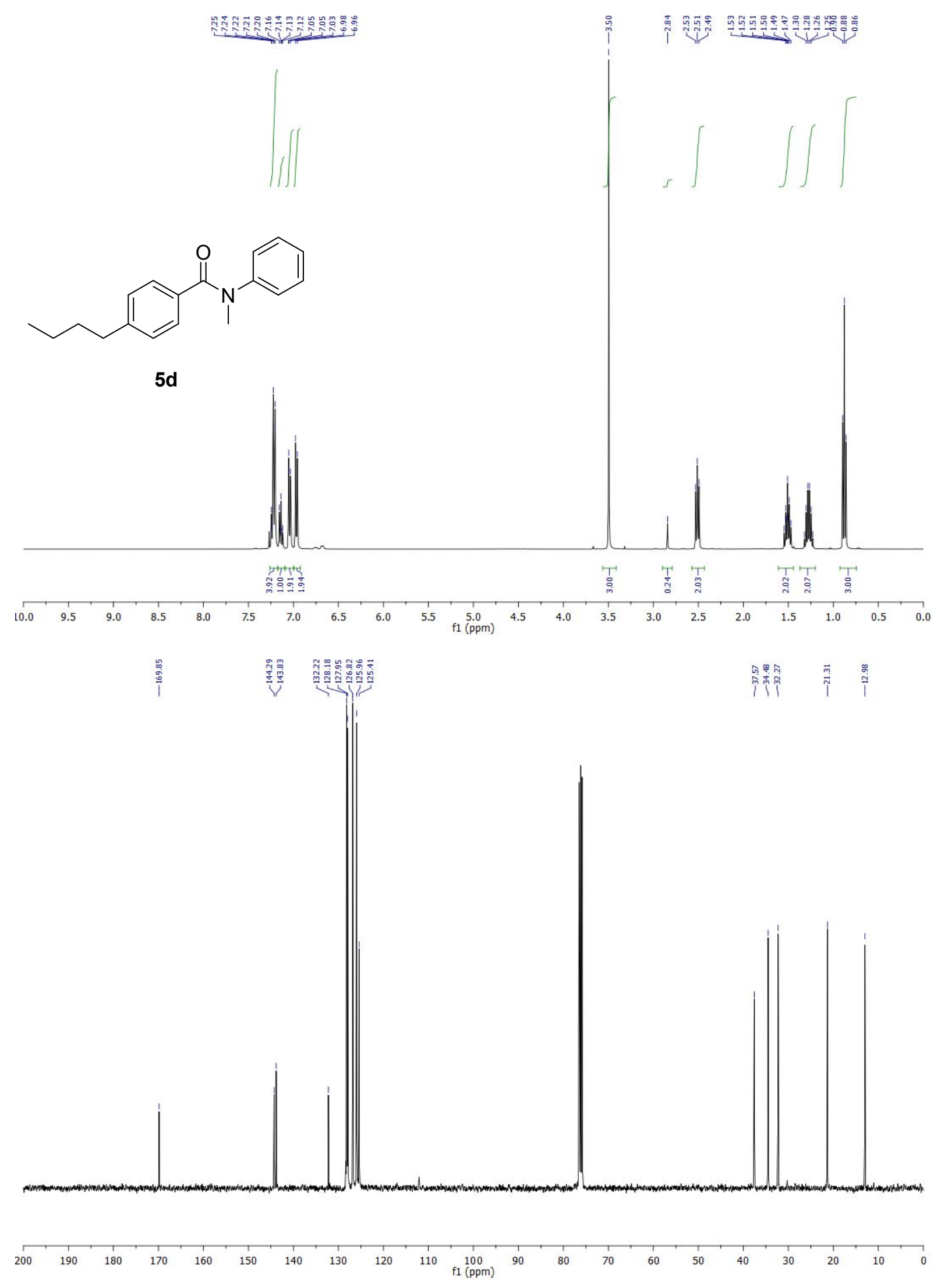

